\title{
Bildgebende Diagnostik bei Pleuraerkrankungen
}

\section{R. C. Bittner}

\section{Diagnostic Imaging in Pleural Diseases}

\section{Zusammenfassung}

Bildgebende Verfahren spielen eine wesentliche Rolle in der Diagnostik und der Therapie pleuraler Erkrankungen. In der Regel ist die Thoraxröntgenaufnahme, heute meist digital angefertigt, unverändert die erste bildgebende Untersuchung. Abhängig von diesem ersten Bildbefund und dem klinischen Aspekt sind weiterführende Untersuchungen die Sonographie, computertomographische Verfahren sowie die Magnetresonanztomographie. Nuklearmedizinische Verfahren spielten praktisch keine Rolle in der Pleuradiagnostik, bis die Positronenemissionstomographie (PET) in die klinische Routine Eingang gefunden hat. Deren Stellenwert in der Diagnostik von Pleuraerkrankungen ist jedoch derzeit noch weitgehend Gegenstand der Forschung.

\section{Abstract}

Imaging plays a major role in the diagnosis and management of pleural diseases. Generally chest radiography remains the initial examination, however, today with digital technique. Depending on first imaging findings and the clinical aspect the diagnostic course may be followed by ultrasound, computed tomography examinations, or magnetic resonance imaging. Radionuclide imaging has not been played a significant role up to now, until positron emission tomography (PET) has gained clinical relevance. However, the role of PET in pleural diseases is not yet defined and scientific works are still in progress.

\section{Pleuraerguss und Pneumothorax}

Der röntgenologische Nachweis eines Pleuraergusses hängt hauptsächlich von der Aufnahmetechnik ab. Bei frontalen Stehendaufnahmen wird pleurale Flüssigkeit überwiegend subpulmonal liegen, was bei mehr als ca. $200 \mathrm{ml}$ zu einer charakteristischen Verrundung des costodiaphragmalen Winkels sowie zu einer Abflachung des medialen Diaphragmaanteils führt [1]. Allerdings können auch auf der Aufnahme in frontaler Ebene bei subpulmonaler Lage $500 \mathrm{ml}$ und mehr ohne röntgenologischen Nachweis bleiben [2]. In der Seitaufnahme und besser noch auf den Aufnahmen in Seitenlage werden schon $20 \mathrm{ml}$ Pleuraerguss sichtbar [3]. Auf Röntgenaufnahmen, die beim liegenden Patien- ten meist als Bettaufnahme in Rückenlage auf Station durchgeführt werden, können selbst große Mengen Pleuraerguss bei freiem Auslaufen und bei gleichzeitig bestehender COPD schwer nachweisbar sein [4].

Insbesondere bei Patienten auf Intensivstationen hat sich die Sonographie als Verfahren zur schnellen und sicheren Diagnostik und Punktion von Pleuraergüssen sowie deren Größenabschätzung etabliert [5-10]. Meist sind pleurale Flüssigkeitsmengen, frei auslaufend oder abgekapselt, als echoarme bzw. -freie Säume zwischen Thoraxwand und Lunge abgrenzbar. Zur Mengenabschätzung dient die Messung der Flüssigkeitsschichtdicke im Bereich der lateralen Thoraxwand beim liegenden Patienten mit 
Tab. 1 Vergleich der sonographisch gemessenen Stärke des Ergusssaumes mit dem wahren Ergussvolumen (nach Punktion/Drainage) (modifiziert nach Eibenberger u. Mitarb. [8] und eigenen Erfahrungen)

\begin{tabular}{|ccc}
\hline $\begin{array}{l}\text { Durchmesser des } \\
\text { Ergusssaumes }(\mathbf{m m})\end{array}$ & $\begin{array}{l}\text { Ergussvolumen } \\
\text { Mittelwert } \\
(\mathbf{m l})\end{array}$ & $\begin{array}{l}\text { Streubreite } \\
(\mathbf{m l})\end{array}$ \\
\hline 0 & 10 & Bis 60 \\
\hline 5 & 70 & $30-150$ \\
\hline 10 & 160 & $80-250$ \\
\hline 15 & 240 & $120-370$ \\
\hline 20 & 350 & $190-600$ \\
\hline 25 & 470 & $320-880$ \\
\hline 30 & 580 & $390-940$ \\
\hline 35 & 700 & $490-1200$ \\
\hline 40 & 900 & $600-1520$ \\
\hline 45 & 980 & $690-1520$ \\
\hline 50 & 1310 & $730-1670$ \\
\hline 55 & 1400 & $800-1800$ \\
\hline$>55$ & 1850 & $910-3200$ \\
\hline
\end{tabular}

leicht angehobener zu untersuchender Seite (Tab. 1) [8]. Pleurale Raumforderungen, z.B. maligne Lymphome, neurogene Tumoren, aber auch Pleuramesotheliome, können im Ultraschall wie Erguss imponieren [7]. In diesen Fällen kann der Einsatz des farb-kodierten Dopplers Fehlbefunde vermeiden durch den Nachweis bzw. Ausschluss perfundierter Gefäße innerhalb des pleuralen Prozesses [11]. Auch die sonographisch gesteuerte Ergusspunktion kann möglicher Bestandteil einer Pleurasonographie sein [6]. Transsudate und Exsudate haben ein oft identisches radiologisches Erscheinungsbild, sonographisch können sie auf der Basis von nachweisbaren Septierungen, die bei ca. 80\% der Exsudate vorliegen, meist unterschieden werden (Abb.1) $[5,7,9,10]$. Gleiches gilt für den Nachweis von echoreichen Anteilen, verdickter angrenzender Pleura und erkennbare Parenchymveränderungen, die deutlich häufiger bei Exsudaten gefunden werden (Abb.1) $[9,10]$.

In CT-Aufnahmen sind Pleuraergüsse sicher nachweisbar, eine sichere Differenzierung von Transsudat und Exsudat gelingt jedoch meist nicht $[12,13]$. Auf der Basis von Dichtewerten kann dagegen eine Einblutung sicher nachgewiesen werden [14,15]. Mitunter erschwert ist die Unterscheidung von Pleuraerguss und Aszites im diaphragmanahen Bereich. Hier hilft die genaue Kenntnis der Schnittbildanatomie, die eine Unterscheidung von (komprimiertem) Lungenparenchym, Pleuraerguss, Zwerchfell, subdiaphragmalem Aszitessaum und Leber- bzw. Milzparenchym-Anschnitt ermöglicht [15-17]. Fehlerhafte Zuordnungen in diesem Bereich beruhen auf Unterlappenatelektasen und nach kaudal gewölbtem Diaphragma z.B. durch subpulmonalen Erguss [18]. Auch können kleine Ergüsse fälschlicherweise für Pleuraverdickungen gehalten werden. Abgekapselte Pleuraergüsse sind mit der CT als linsenförmige Strukturen im Pleurabereich mit flüssigkeitsäquivalenter Dichte, glatter Begrenzung und Verlagerung des angrenzenden Lungenparenchyms identifizierbar (Abb. 2) [1]. Chylöse Flüssigkeitsansammlungen können

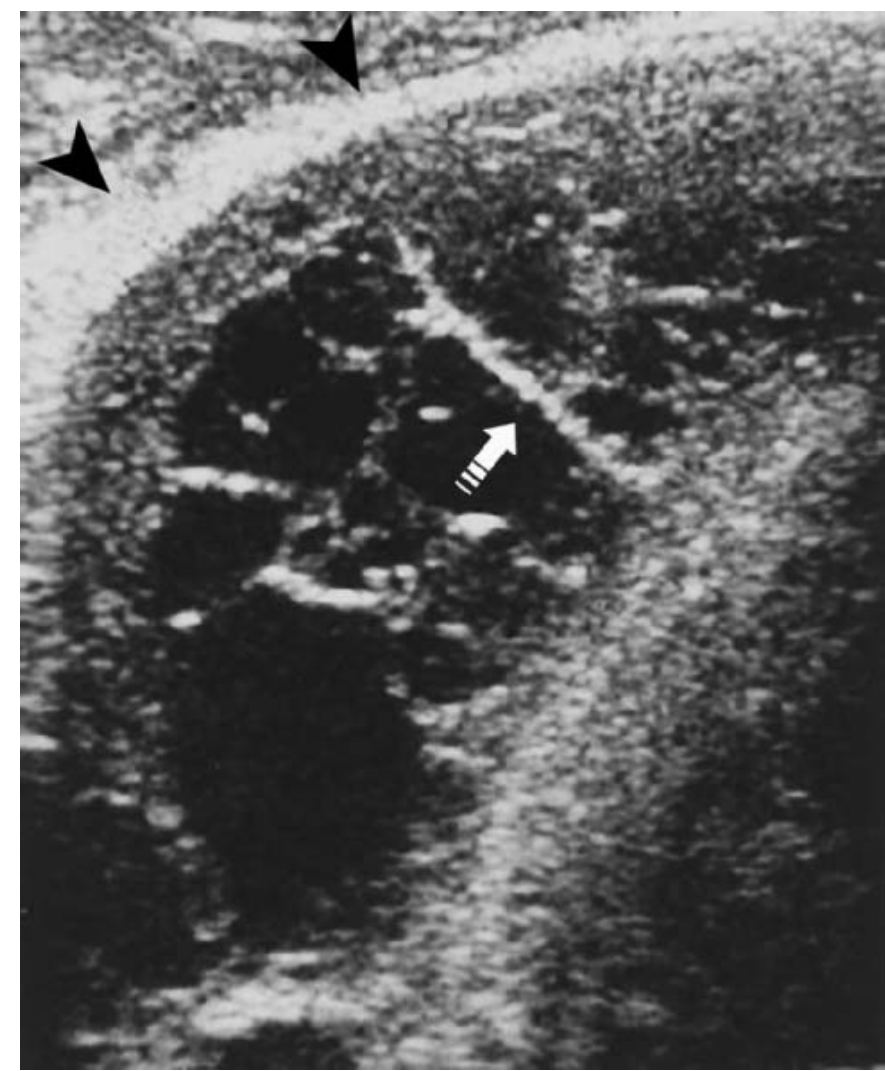

Abb. 1 Exsudativer Erguss. Typische sonographische Darstellung eines exsudativen Ergusses. Multiple Septen innerhalb des Ergusses (Pfeil). Verdickte(s) subpleurales Gewebe und Pleura (Pfeilspitzen).

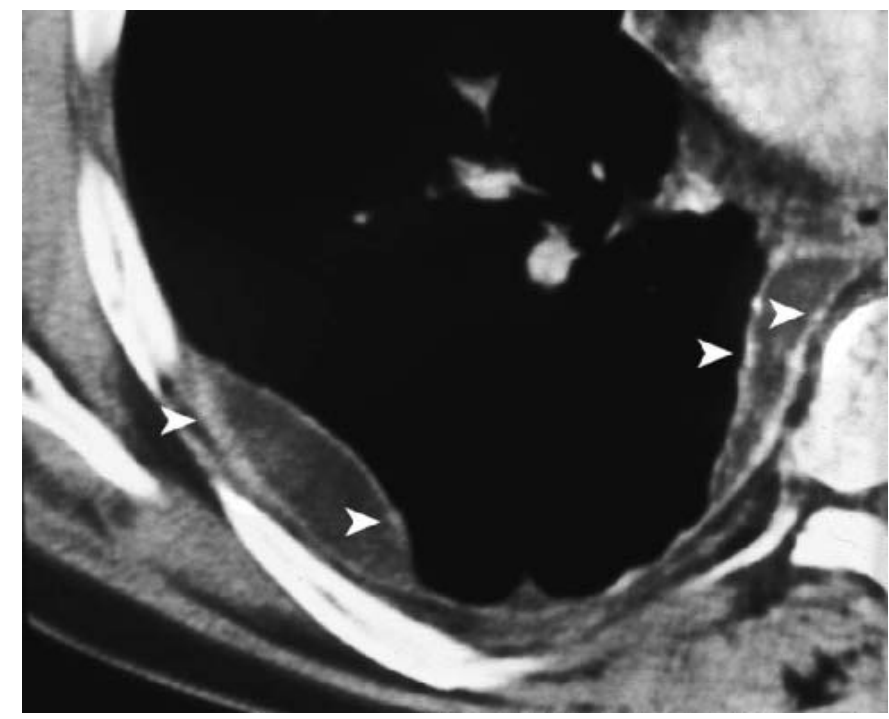

Abb. 2 Pleuraerguss, anteilig abgekapselt, im CT. Nach KM-Gabe deutliche Anreicherung der durch den Erguss aufgeteilten verdickten viszeralen und parietalen Pleurablätter (Pfeilspitzen).

als solche in der Regel nicht auf der Basis von CT-Dichtewerten identifiziert werden [19].

Der Verdacht auf einen malignen Erguss wird auf CT- und MRTAufnahmen nicht selten gestellt durch den gleichzeitigen Nachweis von knotigen bzw. flächigen Pleuraverdickungen mit oder ohne Beteiligung der Thoraxwand (Abb.3) [20,21]. Obwohl eini- 


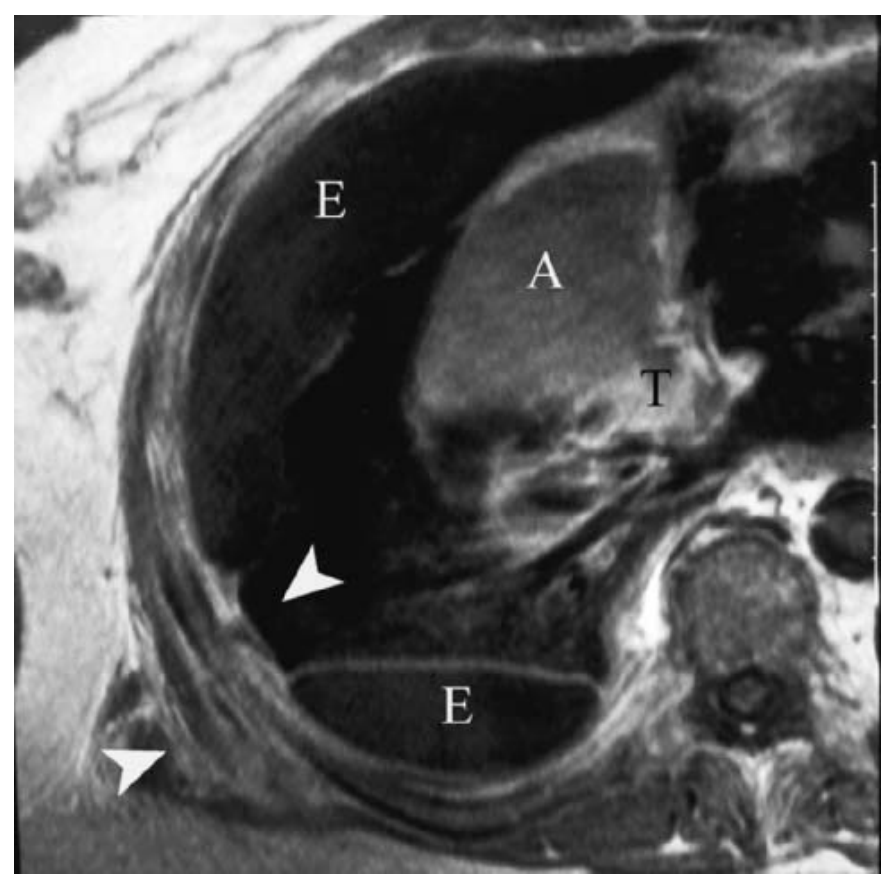

Abb. 3 Maligner Pleuraerguss bei Bronchialkarzinom. T1-gewichtetes MRT nach KM-Gabe. Zentraler Tumor ( $\mathrm{T}$ ) mit Mittellappenatelektase (A). Signalarmer Erguss (E) mit KM-aufnehmenden Pleurablättern. Im laterodorsalen Thoraxwandbereich erkennbare KM-Anreicherung als Zeichen einer tumorösen Infiltration (Pfeilspitzen).

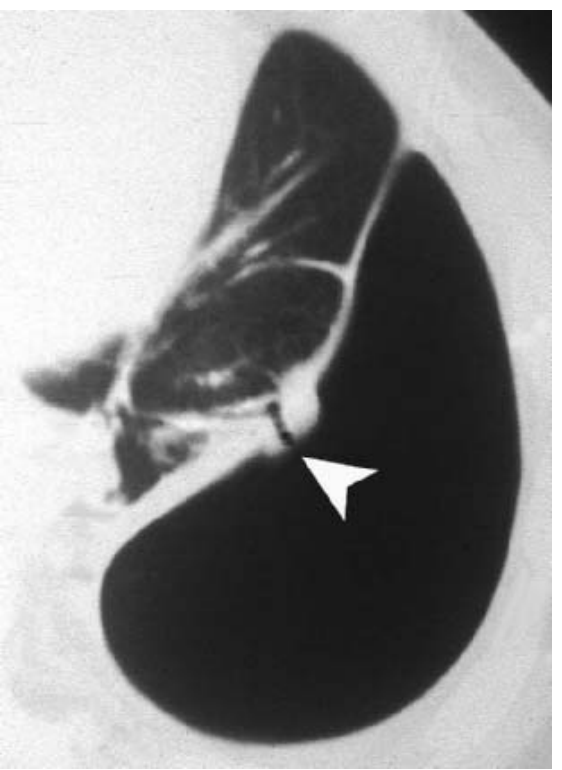

Abb. 4 Bronchopleurale Fistel bei einem Patienten mit schwerer COPD. Das CT im Lungenfenster zeigt eine erkennbare Fistel zwischen Unterlappenbronchialsystem und einem Pneumothorax (Pfeilspitze).

ge Studien gezeigt haben, dass die MRT auf der Basis von Signalintensitäten, Kontrastmittelanreicherung und T2-Relaxationszeiten eine gute Differenzierung von Transsudaten, einfachen Exsudaten, und Exsudaten mit malignen Zellen oder Inflammation ermöglicht, wird dieses aufwendige Verfahren in der klinischen Routine nicht eingesetzt [22]. Auch die verbesserte Differenzierung von Pleuratumoren zu angrenzendem Erguss und Lungenparenchym mit Hilfe der MRT führt nur selten zum klinischen Einsatz [21,23,24].
Der Einsatz der PET bei unklaren pleuralen Erkrankungen mit Erguss zum Ausschluss einer malignen Ursache ist bisher nur in Einzelfällen zu rechtfertigen $(25,26)$. Es gilt, die technisch bedingten Nachteile und Limitierungen des Verfahrens wie begrenzte räumliche Auflösung und Läsionsnachweisgrenze bei ca. $10 \mathrm{~mm}$ gegen mögliches (differenzial)diagnostisches Potenzial unter Berücksichtigung des methodischen Aufwands und der Kosten abzuwägen. Die bisherigen Ergebnisse deuten auf ein hohes differenzialdiagnostisches Potenzial der PET hin.

Pneumothoraces werden meist auf Standard-Röntgenthoraxaufnahmen entdeckt, wobei Exspirationsaufnahmen entgegen der landläufigen Meinung keinen signifikanten Vorteil haben sollen [27]. Schwierig ist der Nachweis von Pneumothoraces bei Liegendaufnahmen. Hier können umschriebene Transparenzerhöhungen hinweisend sein. Auch die Sonographie kann einen Pneumothorax nachweisen [28]. Beim akut traumatisierten oder beatmeten Patient ist der Einsatz der CT zu erwägen, um okkulte Pneumothoraces nachzuweisen. Röntgenologisch kann die einseitige Verlagerung des Diaphragmas nach kaudal einziges Zeichen für einen okkulten Spannungspneumothorax sein. Eine bronchopleurale Fistel kann als Komplikation pulmonaler Infektionen, Operationsfolge, nach Thoraxtraumen und ohne klare Ätiologie auftreten [29]. Typischer Röntgenbefund ist hier ein Seropneumothorax. Mittels Dünnschicht-CT-Aufnahmen lässt sich die Fistel zwischen Bronchialsystem oder Lunge und dem Pleuraraum exakt darstellen (Abb. 4) [14].

Typischerweise präsentiert sich das Empyem auf Röntgenthoraxaufnahmen wie ein (abgekapselter) Pleuraerguss und kann ohne weitergehende Diagnostik nicht von diesem differenziert werden. Empyeme und periphere pulmonale Abszesse lassen sich meist durch die rundliche Form der Abszesse und deren Winkel zur Thoraxwand unterscheiden [29]. Verfahren der Wahl beim Nachweis von Empyemen ist jedoch heute die CT $[13,15,30]$, die gleichzeitig die Möglichkeit der Intervention mit Anlage einer Pleuradrainage unter CT-Durchleuchtung bietet. Von diesem Verfahren wird zunehmend häufig Gebrauch gemacht mit erfolgreicher Drainageapplikation (Abb.5) [31-33]. Zwei CT-Befunde gelten als wegweisend für ein Empyem: nach intravenöser Kontrastmittelgabe kommt es zur Anreicherung der (verdickten) parietalen und viszeralen Pleura und zudem findet sich eine Verdickung des extrapleuralen Weichteilgewebes einschließlich des Fettgewebes in vielen Fällen (Abb. 6) $[13,15]$. Die CT kann relativ sicher zwischen Empyemen und peripheren Lungenabszessen unterscheiden auf der Basis von Befundgrenzen und Verlagerungen von (kontrastierten) Gefäßen und Bronchien [15,34]. Die intravenöse Kontrastmittelapplikation ist in der Regel angezeigt, um eine optimale Differenzialdiagnostik bei pleuralen Flüssigkeitsdepots und assoziierten pleuralen und parenchymatösen Erkrankungen zu gewährleisten (Abb. 6) [34].

\section{Umschriebene Pleuraerkrankungen}

Die häufigsten lokal begrenzten Pleuraveränderungen sind Pleuraplaques, umschriebene Pleuratumoren und eine begrenzte Invasion pulmonaler Raumforderungen. Pleuraplaques sind meist zwischen einem und zehn Millimeter dick, imponieren als umschriebene, oft verkalkte fibröse Pleuraauflagerungen und gelten 


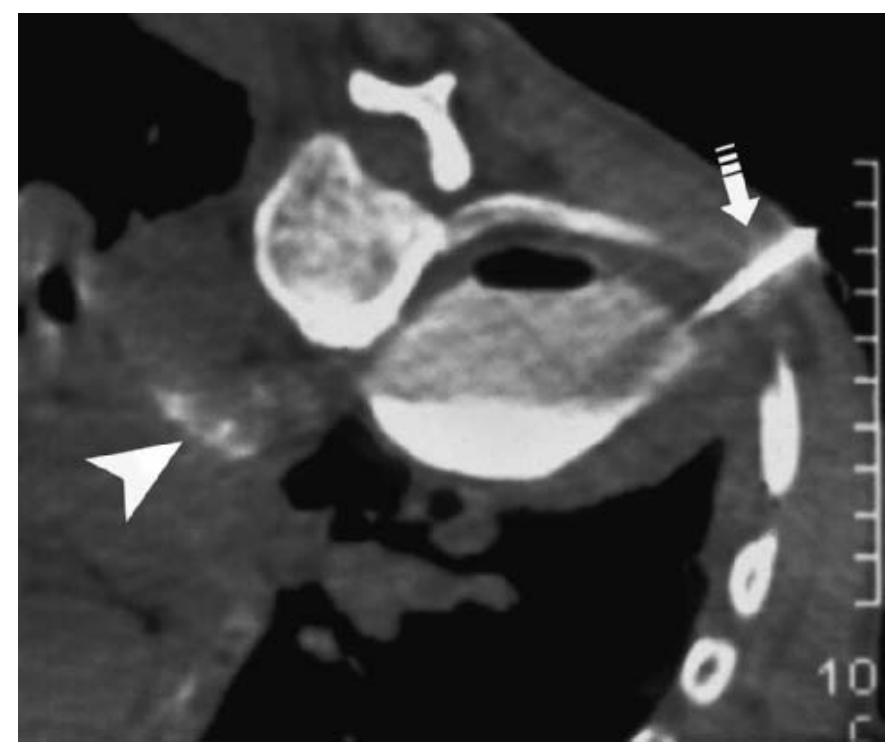

Abb. 5 Durchleuchtungs-CT-Aufnahme mit reduzierter Matrix, CTgestützte Drainageanlage (Pfeil) in ein Pleuraempyem. KM-Füllung der Empyemhöhle nach Ösophaguskontrastierung als Nachweis einer Nahtinsuffizienz nach Ösophagusruptur mit Ausbildung einer ösophago-pleuralen Fistel und Mediastinitis (Pfeilspitze).

insbesondere bei Tafelberg-artiger Konfiguration als pathognomonisch für eine pleurale Asbestose [35] (Abb. 7). Posterolaterale Plaques von mindestens $5 \mathrm{~mm}$ Dicke und bilaterale verkalkte diaphragmale Plaques sind nahezu $100 \%$ spezifisch für eine Asbest-assoziierte Pleuraerkrankung [36]. In Regionen mit einer hohen Prävalenz für Tuberkulose muss differenzialdiagnostisch diese Erkrankung mitbedacht werden. Die Sensitivität für Pleuraplaques auf der konventionellen Thoraxröntgenaufnahme erreicht nur ca. 80\% [36]. Digitale Aufnahmen können durch Nachbearbeitung wie Kantenanhebung oder Histogramm-Modifizierung den Nachweis für Pleuraplaques verbessern [37]. Der sonographische Nachweis von Pleuraplaques gelingt sicher erst ab Dicken größer $3 \mathrm{~mm}$ und besser bei begleitendem Pleuraerguss $[7,10]$.

Die High-Resolution (HR-) CT, bis vor ca. 3 Jahren als Einzelschicht-Verfahren und heute meist als Mehrzeilen-Spiral-CT durchgeführt, ist mit einer Schichtdicke von 1-2 mm und kantenbetontem Rekonstruktions-Algorithmus die Methode der Wahl zum Nachweis pleuraler Plaques [35,36,38-40]. Diese können auf HR-CT-Aufnahmen erkannt werden als umschriebene Areale pleuraler Verdickung, die sich nach peripher von der angrenzenden Rippe und dem extrapleuralen Weichteilgewebe durch eine dünne Fettlamelle abgrenzen lassen (Abb. 8a) [41]. Hauptsächlich aufgrund der Strahlenexposition und nicht zuletzt aus Kostengründen wird die HR-CT nicht routinemäßig als Suchverfahren für pleurale Erkrankungen eingesetzt, hat diesbezüglich aber bei Niedrigdosis-Technik und sinkenden Kosten enormes diagnostisches Potenzial. Bei unklaren röntgenologischen Befunden, z. B. bei der Differenzierung pulmonaler versus pleuraler Herdbefunde und zum Ausschluss falsch-positiver Röntgenbefunde wird die HR-CT bereits erfolgreich eingesetzt $[15,35,36]$.

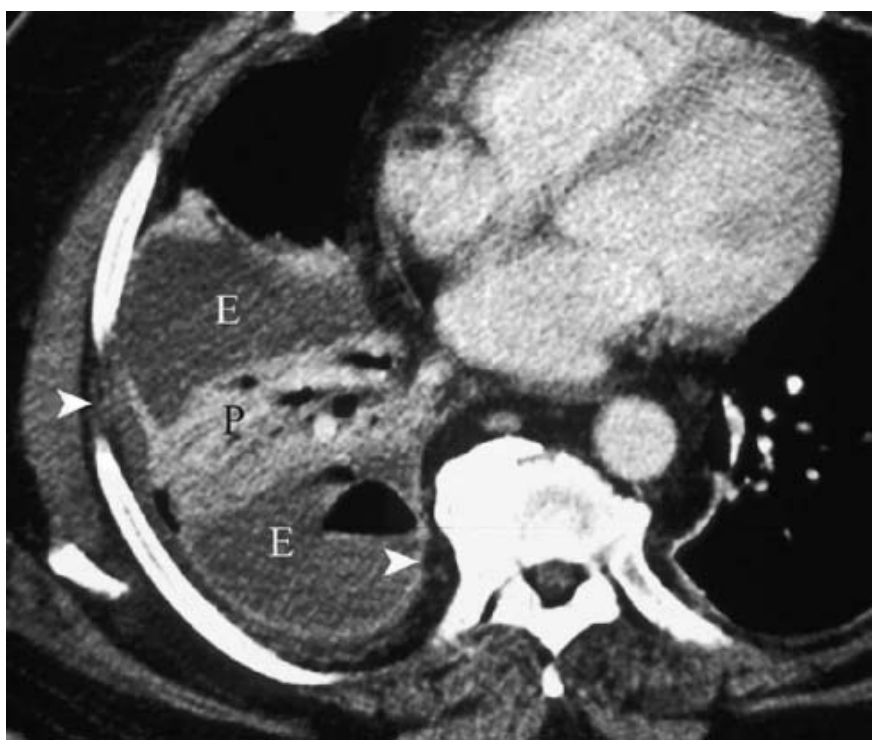

Abb. 6 Chronisches Pleuraempyem im KM-CT. Relativ dichte Empyemanteile (E) mit dazwischen liegendem komprimierten Lungenparenchym $(P)$, Lufteinschlüsse im dorsalen Bereich. Verdicktes subpleurales Weichteilgewebe angrenzend an die verdickte kontrastierte Pleura (Pfeilspitzen).

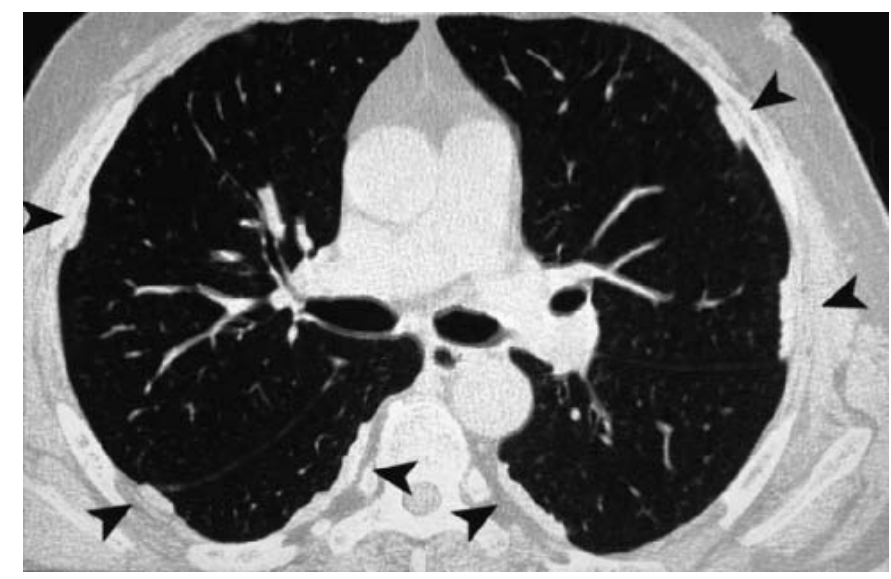

Abb. 7 1-mm-HR-CT-Aufnahme im Pleurafenster bei bekannter pleuraler Asbestose. Gut abgrenzbare charakteristische anteilig verkalkte Tafelberg-artige Plaques mit bis zu 8 mm Dicke (Pfeilspitzen).

Der Nachweis pleuraler Plaques gelingt auch mit der MRT, die Sensitivität ist der der HR-CT aber unterlegen [24,42]. Die typischen Verkalkungen imponieren meist als Signalaussparungen in Standard-MRT-Aufnahmen (Abb. 8b) [42]. Bei zunehmender Verdickung oder Vergrößerung bekannter pleuraler Plaques kann die hochauflösende (HR-) MRT diagnostische Zusatzinformationen geben (Abb. 9a, b) [24].

Die Anreicherungen des Radionuklids in Pleuraplaques bei Indium-111-Pentreotid-Szintigraphien haben sich leider als unspezifisch herausgestellt und können zu falsch-positiver NeoplasieDiagnose bei Patienten mit benignen pleuralen Plaques führen [43]. Zur Wertigkeit der PET bei Pleuraplaques gibt es bis heute keine aussagekräftigen Untersuchungsergebnisse [25]. 

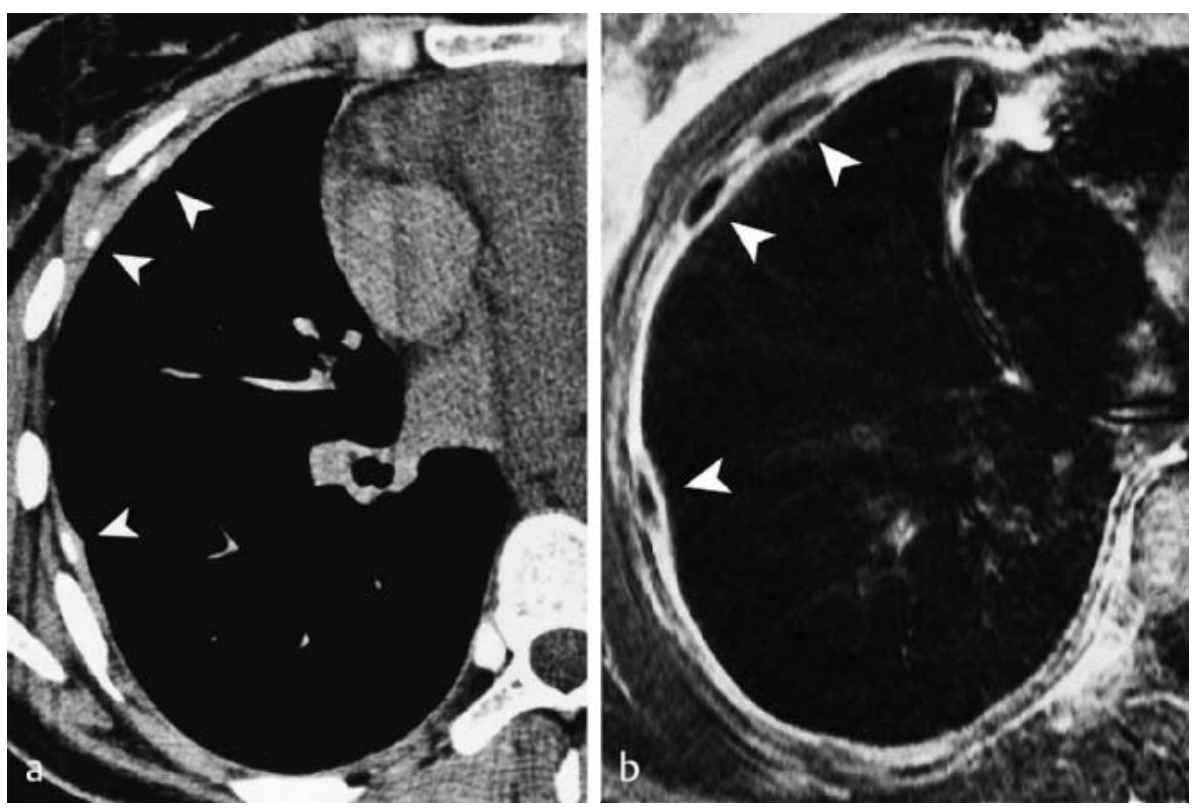

Abb. 8a, b a Native 1-mm-HR-CT-Aufnahme im Weichteilgewebefenster, Pleuraasbestose nach umweltbedingter Exposition in der Türkei. Mehrere teilweise kalkhaltige Plaques erkennbar, die subpleurale Fettgewebslamelle ist gut abgrenzbar (Pfeilspitzen). b Vergleichbare MRT-Aufnahme des gleichen Patienten, T1-gewichtet nach KMGabe. Signalfreie Darstellung der kalkhaltigen Plaque-Anteile (Pfeilspitzen) bei intensiver zirkumferenter KM-Aufnahme der verdickten Pleura.
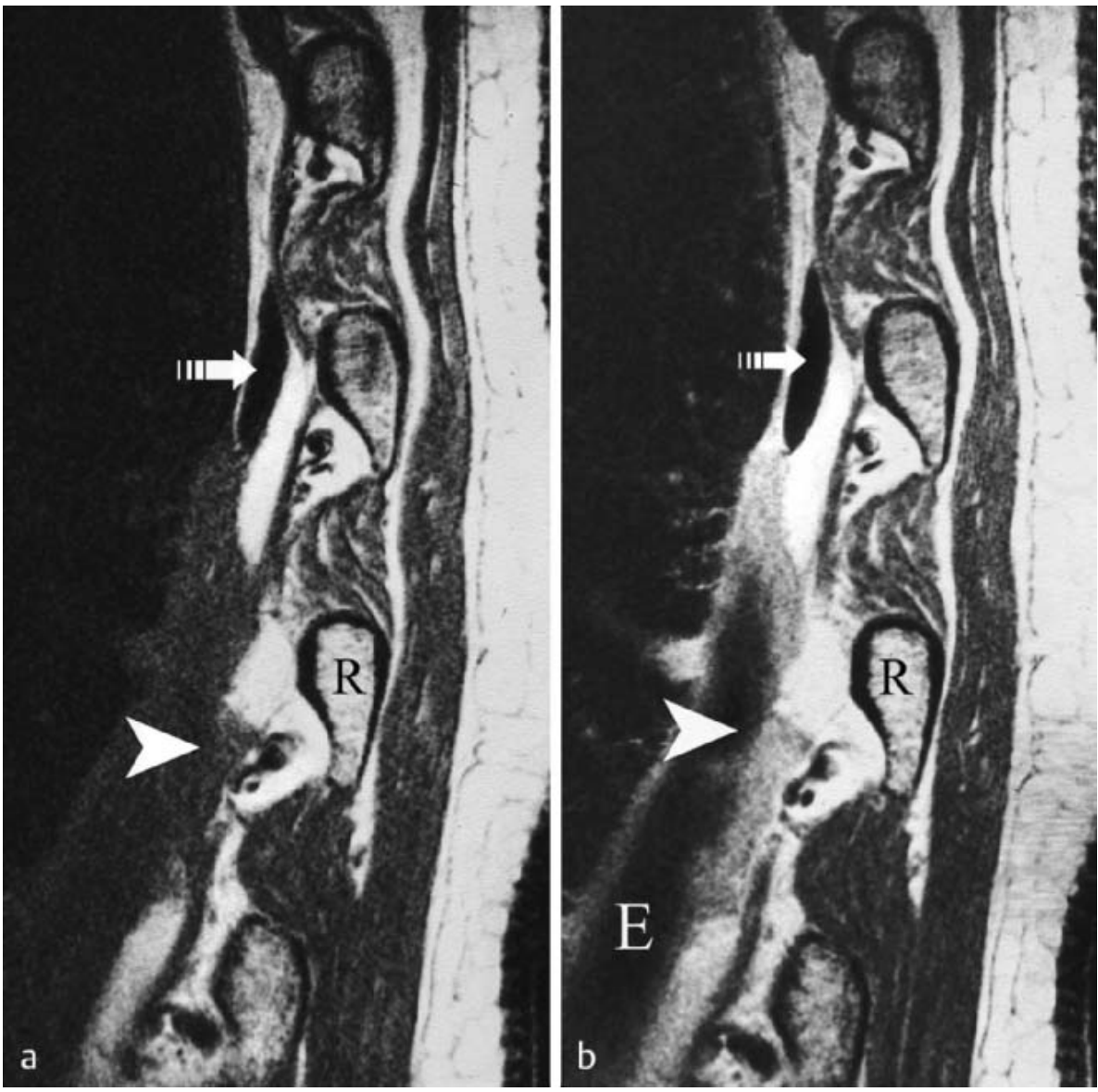

Abb. 9a, b Patient mit bekannter Pleuraasbestose und größenprogredientem Plaque. Hochaufgelöste sagittale T1-gewichtete MRT-Aufnahmen vor und nach KM durch die Thoraxwand in diesem Bereich. Signalfreier verkalkter Plaque (Pfeil). Rippe $(R)$. a Weichteiläquivalentes Gewebe mit Übergreifen auf das Fettgewebe des Interkostalraumes (Pfeilspitze). b Deutliche KMAufnahme des infiltrierenden Gewebes. Operative Sicherung eines lokal infiltrierenden malignen Pleuramesothelioms.

Rundatelektasen sind keine primär pleuralen Veränderungen, treten aber häufig im Zusammenhang mit Asbest-assoziierten Pleuraerkrankungen auf $[14,35,38,44,45]$. Als ursächlich wird ein exsudativer Pleuraerguss angesehen, der zu pleuralen Adhäsionen und nachfolgend zu einer Einfaltung und Fixierung von angrenzendem Lungenparenchym führt. Die posterioren medialen Unterlappen sind bevorzugt betroffen. Das atelektatische Parenchym kann eine pleurale oder pulmonale Neoplasie vor- täuschen [45]. Eine angrenzende Pleuraverdickung findet sich fast immer, der typische „Kometenschweif“ der Rundatelektase im Röntgenbild repräsentiert den bogenförmigen Verlauf der zuführenden Bronchien und Gefäße [35,45]. Die sonographischen, CT- und MRT-Befunde bei einer Rundatelektase zeigen eine bis zu $7 \mathrm{~cm}$ große rundliche Raumforderung mit angrenzender Pleuraverdickung (Abb. 10a, b) [21,45-48]. Das wesentliche sonographische Kriterium für eine Rundatelektase ist eine stark echorei- 

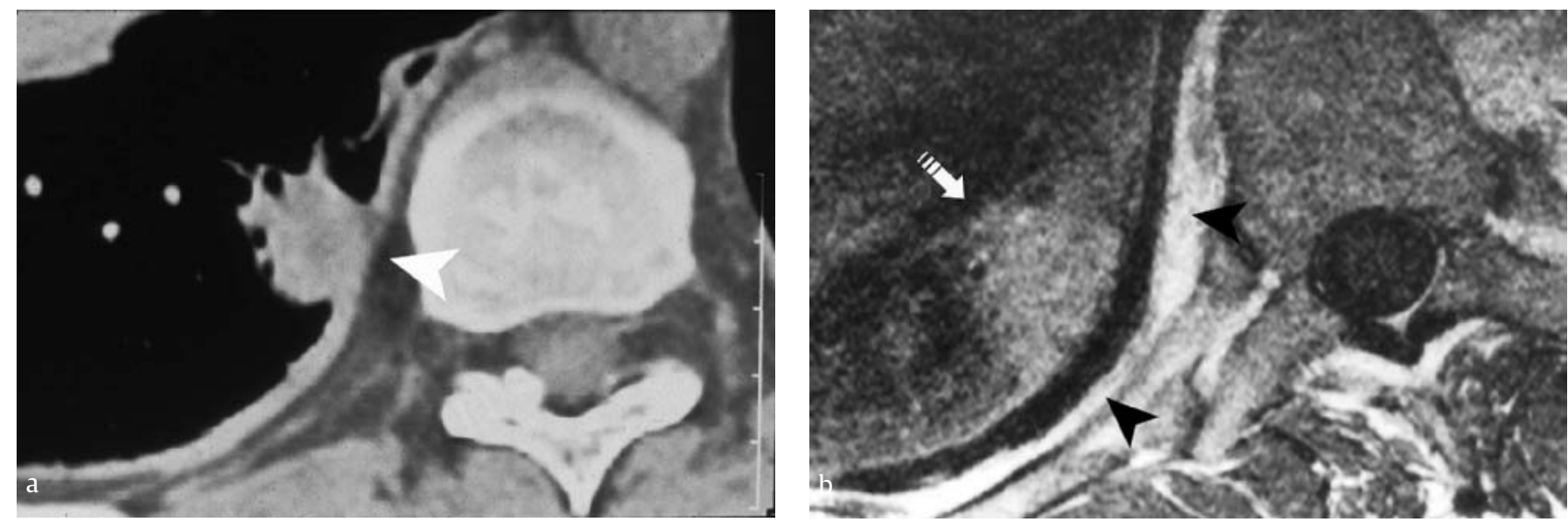

Abb. 10a, b Rundatelektase. a Weichteildichte rundliche Struktur mit kleinen Ausziehungen in Richtung Hilus und breitem Kontakt zur verdickten Pleura (Pfeilspitze), im CT suspekt auf Rundatelektase. b Korrespondierendes T1-gewichtetes MRT nach KM zeigt ein typisches parenchymales Anreicherungsmuster (Pfeil). Die rindenartig verdickte Pleura stellt sich nahezu signalfrei dar (Pfeilspitzen).

che bandförmige Struktur, die von der Pleuraoberfläche bis in die Raumforderung zu verfolgen ist [46]. Dieser Befund soll mit der verdickten eingefalteten Pleura korrelieren. Die KM-unterstützte CT zeigt einen deutlichen, meist homogenen Dichteanstieg der Rundatelektase im Vergleich zur in der Regel weniger starken KM-Anreicherung einer Neoplasie [14,23,44,45]. Die MRT zeigt meist eine homogene Raumforderung mit Kontakt zur verdickten Pleura und niedriger Signalintensität auf den nativen T1-gewichteten Aufnahmen, nach KM-Gabe kommt es zu einer typischen parenchymatösen Anreicherung (Abb. 9b). Die Signalintensität auf den T2-gewichteten Aufnahmen variiert von niedrig bis hoch $[21,23,42,47,48]$.

$\mathrm{Zu}$ den umschriebenen pleuralen Tumoren gehören Fibrome, Lipome, Liposarkome und die lokalisierte Infiltration der Pleura durch angrenzende Tumoren, meist Bronchialkarzinome. Maligne Mesotheliome und Pleurametastasen zeigen dagegen überwiegend eine diffuse flächige Ausbreitung. Solitäre Fibrome und Lipome als die häufigsten benignen Tumoren stellen sich röntgenologisch als glatt berandete, gut abgrenzbare rundliche und gelegentlich lobulierte Tumoren mit einem Durchmesser zwischen einem und $40 \mathrm{~cm}$ dar [49]. Man findet meist einen stumpfen Winkel mit der Thoraxwand oder dem Mediastinum, die Verdrängung bzw. Kompression angrenzenden Lungenparenchyms ist typisch. Gestielte Fibrome haben einen Anteil von bis zu $50 \%$, sind charakteristischerweise lageveränderlich unter Durchleuchtung, was als der sicherste Hinweis auf eine benigne Genese gilt [49]. Die Sonographie wird bei solitären pleuralen Tumoren oft eingesetzt, um Biopsien zu steuern (Abb.11) [50]. Eine einzelne Studie berichtet über differenzialdiagnostisches Potenzial von Doppler- und farbkodierter Sonographie bei benignen und malignen pleuraständigen Tumoren auf der Basis vaskulärer Strömungssignale [11].

In der CT zeigen sich lokalisierte homogene, glatt begrenzte Weichteiltumoren mit engem Bezug zu Pleura oder Fissuren (Abb. 12a) [15,23]. Das KM-Anreicherungsverhalten ist variabel, kann bei zentraler Nekrose oder größeren Binnenverkalkungen auch deutlich inhomogen sein. Gelegentlich kann ein begleitender Pleuraerguss die Abgrenzbarkeit des Tumors erschweren

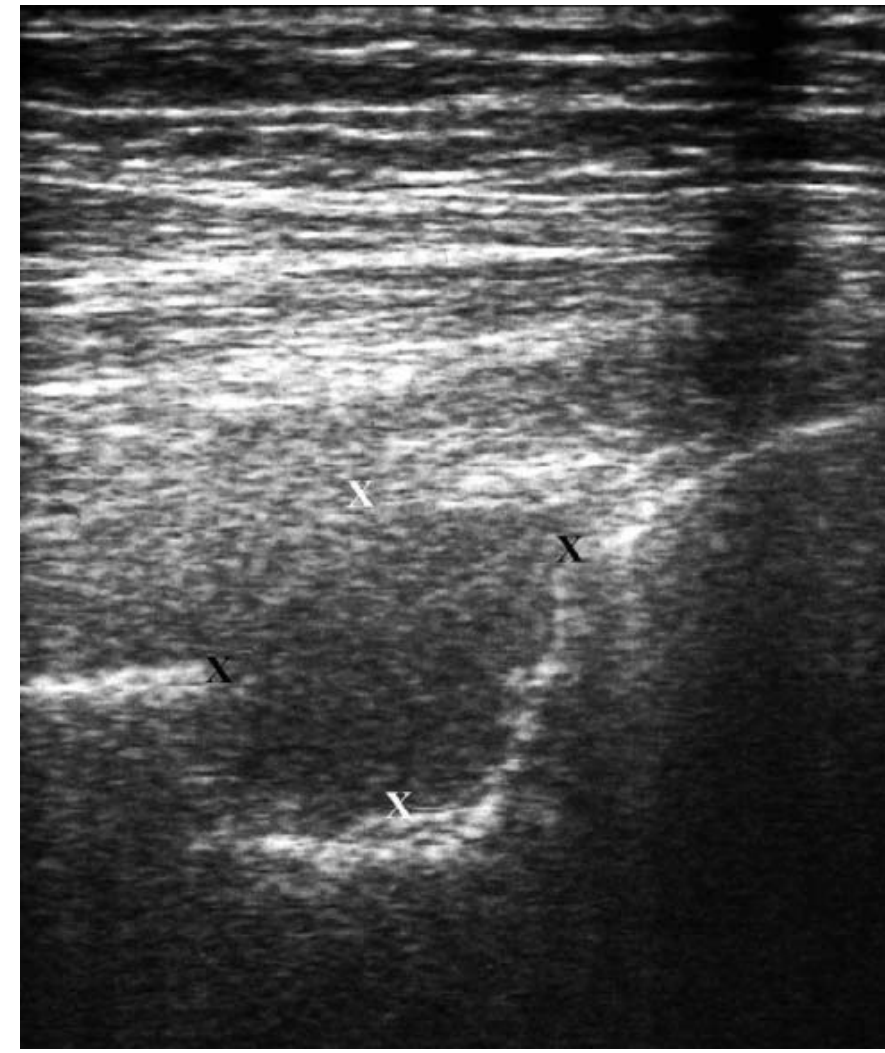

Abb. 11 Malignes fibröses Histiozytom der Pleura. Sonographisch gut identifizierbare Raumforderung mit Unterbrechung der Grenzlinie Pleura-Lunge als Hinweis auf Invasion der inneren Thoraxwand. Markierung zur präzisen Größenmessung, bei umschriebenen Tumoren gut möglich. Gefäßbeurteilung durch Zuschalten des Dopplermodus.

$[15,23]$. Der Nachweis von indirekten Zeichen wie Kompressionsatelektase oder Bronchien- und Gefäßverlagerungen erlauben in der Regel eine sichere Differenzierung zwischen pleuraler und parenchymatöser Raumforderung in der CT $[23,51,52]$. Die Winkel zwischen Tumor und Thoraxwand hängen ab von der Größe des Tumors, kleine Tumoren zeigen eher stumpfe Winkel, größere Tumoren eher spitze Winkel. Das einzige verlässliche CT-Kriterium zur Differenzierung maligner von benignen Pleura- 

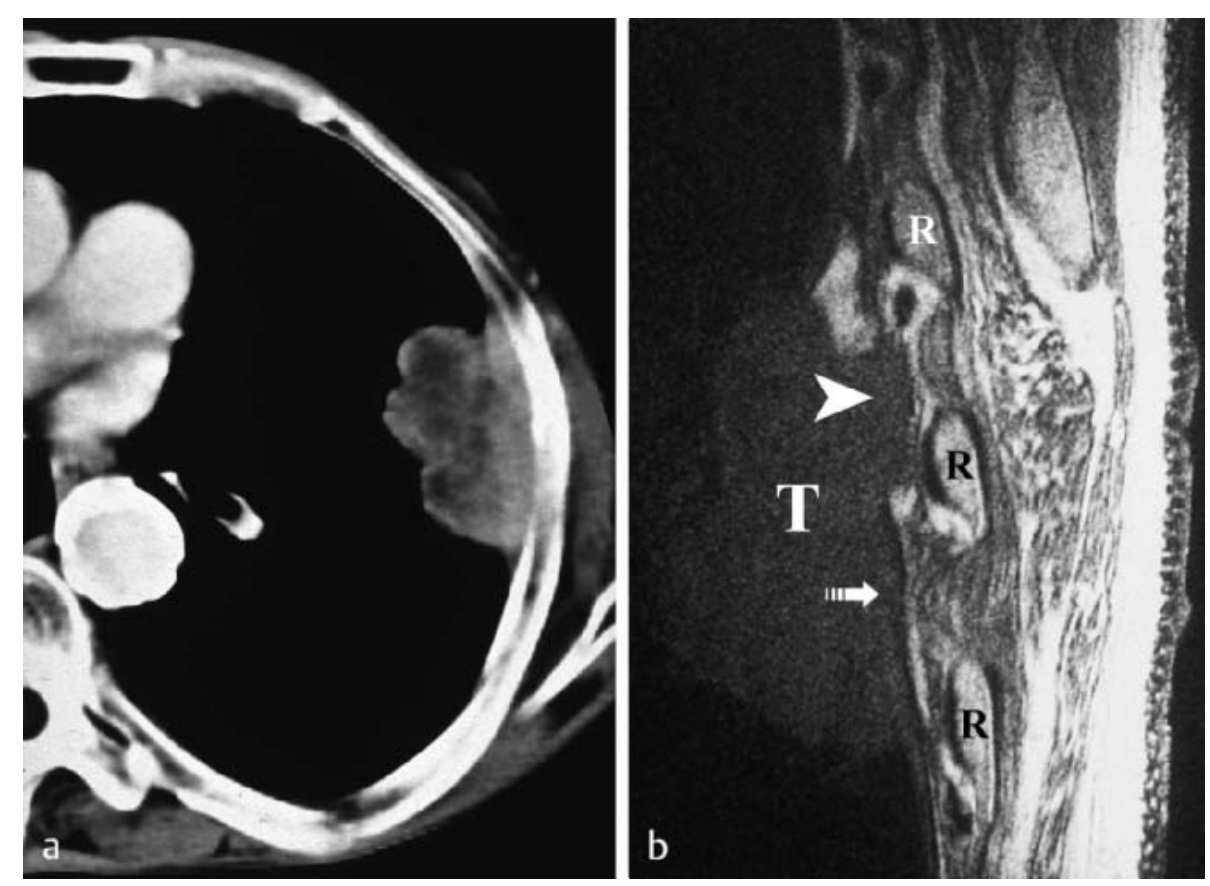

Abb. 12a, b Malignes fibröses Histiozytom der Pleura. a Polypös konfigurierter breitbasig pleural aufsitzender Tumor mit inhomogener Anreicherung im KM-CT. b Gleiche Raumforderung in T1-gewichteter hochaufgelöster MRT der Thoraxwand, sagittaler Schnitt. Umschriebene Infiltration in die innere Thoraxwand (Pfeilspitze), weiter kaudal noch intakte Pleura mit subpleuralem Fettgewebe (Pfeil). Rippen (R). Tumor (T).

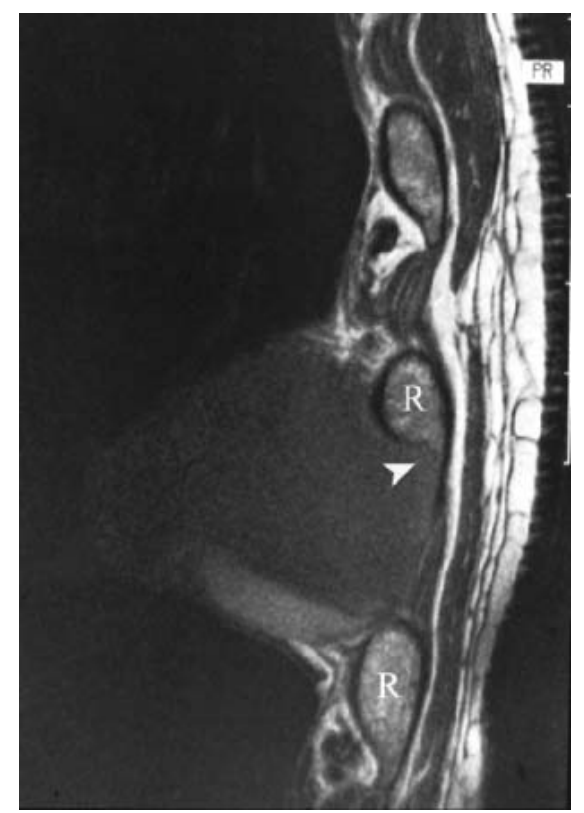

Abb. 13 Neurofibrom. Natives T1-gewichtetes hochaufgelöstes sagittales MRT. Druckarrosion der mittleren Rippe deutlich erkennbar durch fehlende Kortikalis (Pfeilspitze). Rippen (R).

tumoren ist jedoch der Nachweis der Tumorinvasion in die angrenzende Thoraxwand mit oder ohne Rippendestruktion [21, 24,53-56].

Im Vergleich zur CT erlaubt die MRT eine deutlich überlegene Weichteilgewebe-Kontrastauflösung, gut auf den T2-gewichteten und besser auf den T1-gewicheten Aufnahmen vor und nach KM-Gabe [21,24,57 -60]. Da pleurale Veränderungen meist relativ nahe der Hautoberfläche liegen, bieten sich Oberflächenspulen in der MRT für eine verbesserte Darstellung von Pleuratumoren an (Abb. 12b) [24]. (Neuro-)Fibrome zeigen in der MRT in den T2-gewichteten Aufnahmen oft eine inhomogene, aber überwiegend niedrige Signalintensität, während in den T1-gewichteten Aufnahmen die Signalintensität eher Muskel-äquivalent homogen ist (Abb.13) [56,59-62]. Areale höherer Signalintensität entsprechen Nekrosezonen oder myxoider Veränderung. Nach
KM-Gabe findet sich meist eine deutliche, auch inhomogene Anreicherung entsprechend der Vaskularisation der Raumforderung. Die Differenzierung von Druckarrosionen angrenzender Rippen von tumoröser Infiltration gelingt mit der hochauflösenden MRT am besten (Abb. 13) [56,59-62].

Benigne Lipome sind überwiegend asymptomatisch und werden meist als Zufallsbefunde auf Röntgenthoraxaufnahmen entdeckt [63]. Sie können intrathorakal oder transmural lokalisiert sein, mit intra- und/oder extrathorakalem Anteil, die Mehrzahl findet man im Bereich der lateralen Thoraxwand. Die Diagnose kann zuverlässig mit der CT gestellt werden durch den Nachweis fettäquivalenter Dichtewerte mit gelegentlich bindegewebig-septaler Binnenstruktur und einzelnen Verkalkungen nach Fettgewebsnekrosen. Das angrenzende Lungenparenchym wird verlagert. Gelegentlich findet man eine Pseudokapsel [64]. Bei inhomogener Binnenstruktur und fettäquivalenten Dichtewerten, unregelmäßiger Kontur und Begleiterguss sowie KM-aufnehmenden Tumoranteilen muss ein Liposarkom ausgeschlossen werden (Abb. 14a) [65]. Der Verdacht auf ein pleurales Liposarkom in der MRT gründet sich auf die gleichen vorgenannten Kriterien wie in der CT, der Nachweis bizarr konfigurierter KM-aufnehmender Tumoranteile gelingt durch den überlegenen Weichteilgewebekontrast in der MRT jedoch besser, zudem liegt meist ein diffuser pleuraler Befall nach radiologischen Kriterien vor (Abb. 14b) [24].

Die direkte multiplanare Abbildung der (hochauflösenden) MRT erlaubt die sichere Festlegung auf die pleurale Genese einer Raumforderung, wenn dies in Röntgenaufnahmen oder in der CT nicht gelingt. Die Vorteile der MRT werden insbesondere deutlich bei Lage des Tumors im Bereich der Lungenspitze oder in Höhe des Zwerchfells. Hier können sagittale oder koronare MRT-Schnittbilder exakt den Tumor in Relation zum angrenzenden Plexus brachialis oder zu den Zwerchfellschenkeln darstellen (Abb.15, Abb.16) [24,59]. 

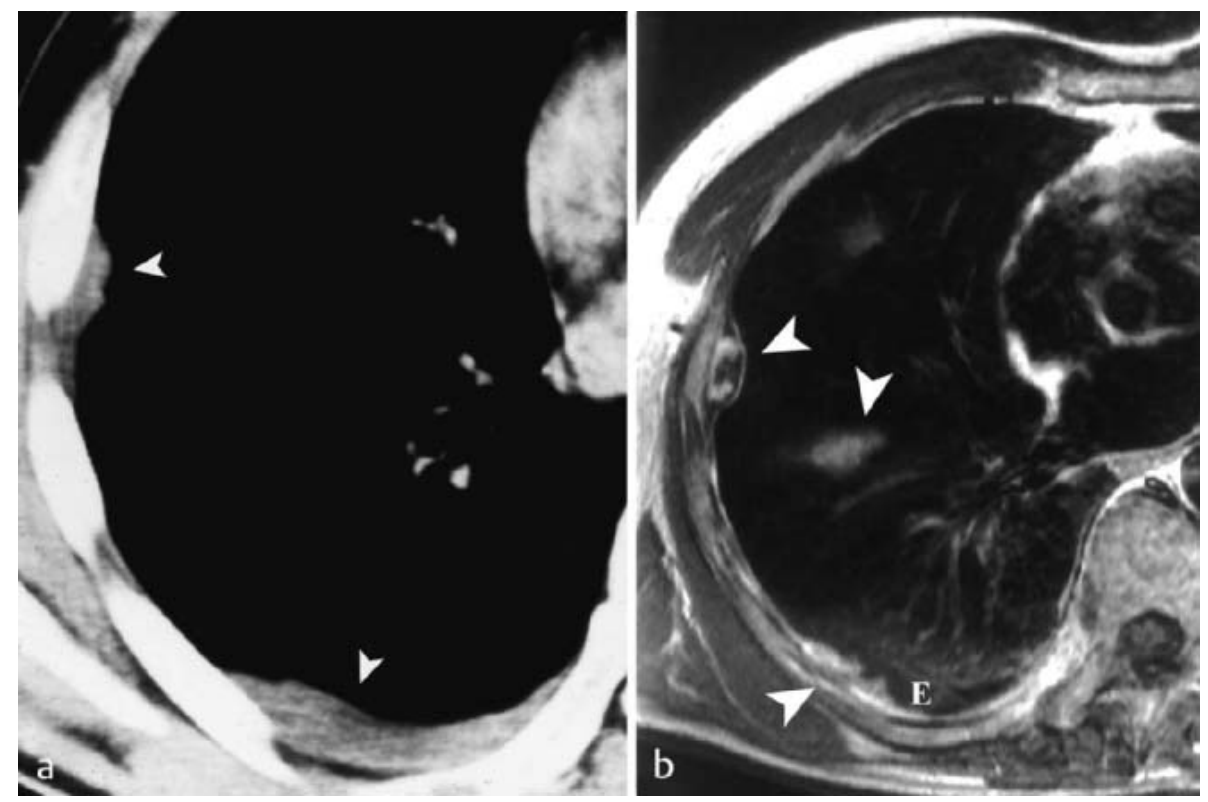

Abb. 14a, b Primär pleurales Liposarkom. a Das KM-CT zeigt pleurale Veränderungen ohne weitergehende Differenzierung, erschwerte Abgrenzung von Erguss und Pleuraverdickung. b Korrespondierendes T1-gewichtetes KM-MRT. Überlegene Weichteilgewebe-Kontrastauflösung mit Kontrastierung des irregulär konfigurierten pleuralen Tumorgewebes bis in die Fissur (Pfeilspitzen) und Abgrenzbarkeit des kleinen Pleuraergusses dorsal (E).

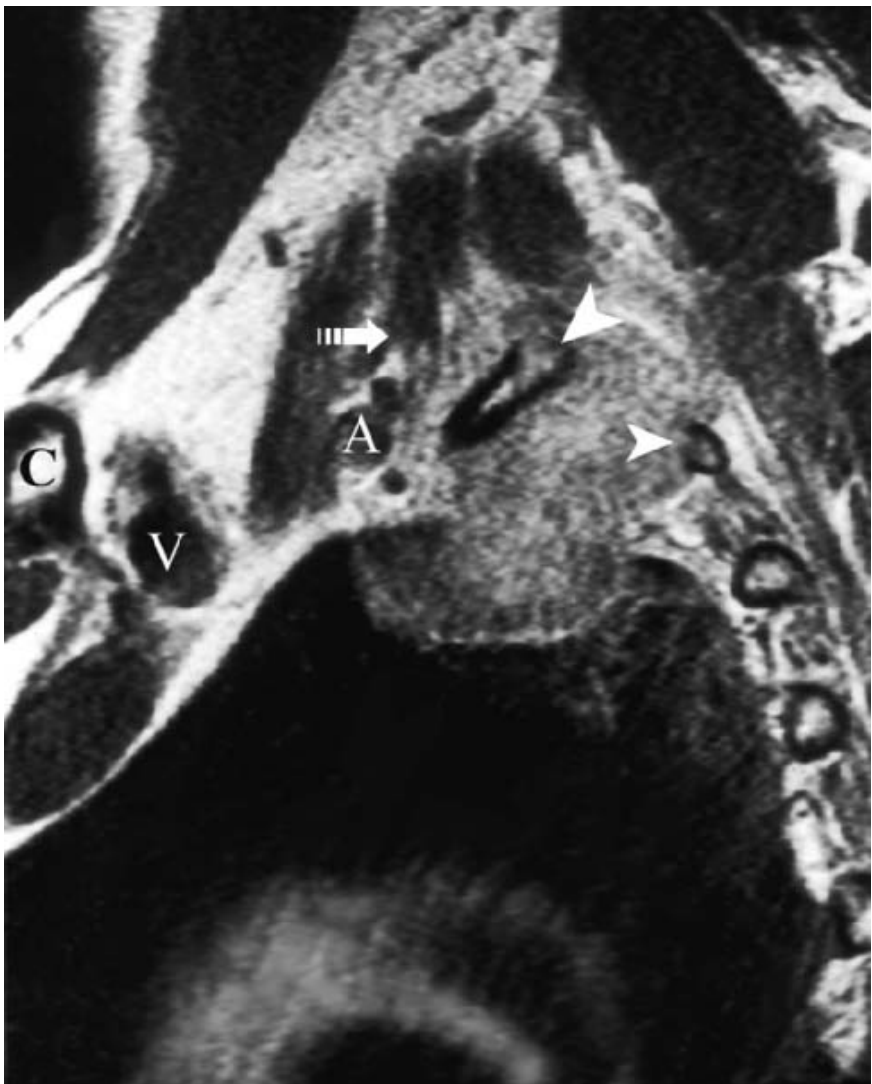

Abb. 15 Bronchialkarzinom vom Pancoast-Typ. Exzellente Darstellung der Ausbreitung des Tumorgewebes im T1-gewichteten sagittalen MRT der Thoraxapertur. Rippenarrosionen durch unterbrochene Kortikalis (Pfeilspitzen), der Tumor grenzt direkt an die Faszikel des Plexus brachialis (Pfeil). Clavicula (C), Vena (V) und Arteria (A) subclavia links.

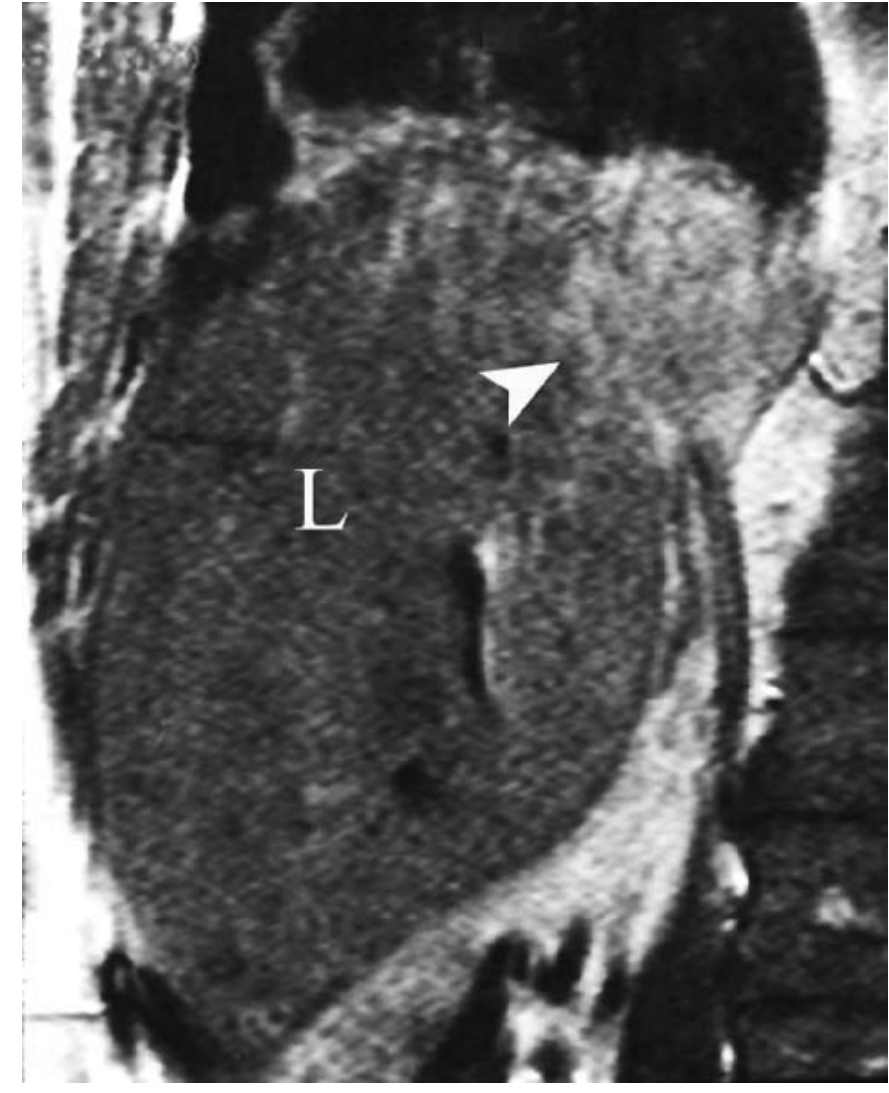

Abb. 16 Primär pleurales Liposarkom. Koronales T1-gewichtetes KMMRT, Übergreifen des inhomogen kontrastierten Tumorgewebes auf das Leberparenchym (L). Infiltration des medialen Zwerchfellanteils mit Abbruch der Zwerchfellkontur (Pfeilspitze).
Der Stellenwert der PET bei solitären pleuralen Tumoren ist bisher nicht ausreichend evaluiert $[25,66,67]$. In Einzelfällen hat die PET ihr differenzialdiagnostisches Potenzial angedeutet durch den Nachweis einer signifikant höheren Anreicherung in malignen Tumoren im Vergleich zu der in benignen Raumforderungen $[66,67]$.
Die häufigste Ursache einer malignen pleuralen Erkrankung ist das Bronchialkarzinom in ca. 50\% aller Fälle. Die umschriebene Tumorinfiltration der Pleura verändert das Staging: während die Invasion der viszeralen Pleura T2 entspricht, führt eine mediastinale oder parietale Pleurainvasion zum T3, gleiches gilt für Tumorwachstum in den Sulcus superior und die Thoraxwand. 
Der Nachweis einer Tumorinvasion von mediastinalen Organen oder von Wirbelkörpern bzw. Sternum sowie des Diaphragmas im Sinne eines T4-Stadiums würde sowohl erhebliche prognostische Relevanz als auch Auswirkungen auf die Therapie haben [68]. Bei Nachweis einer pleuralen bzw. pleuraständigen Raumforderung auf Röntgenaufnahmen eines Patienten mit Bronchialkarzinom wird häufig die Sonographie zur weiterführenden Diagnostik eingesetzt (Abb.17a). Die CT tut sich nicht selten schwer, eine Angrenzung eines Tumors an Pleura, Mediastinum oder Diaphragma von einer Invasion zu differenzieren (Abb. 17b) $[24,59,69,70]$. Das Ausmaß des Kontaktes von Tumor und Pleura, pleurale Verdickung und Pleuraerguss haben eine niedrige Spezifität für den Nachweis einer Pleurainvasion. Einige Autoren schlagen deshalb vor, mittels eines iatrogenen Pneumothorax und einer anschließenden CT oder MRT eine Tumorinfiltration über die Pleura viszeralis hinaus nachzuweisen [71]. Die MRT ist der CT im Nachweis einer Tumorinvasion der Thoraxwand sowie der kostalen und mediastinalen Pleura überlegen $[21,24,59,72-75]$. Dabei zeigen sich in der Regel einer oder mehrere der folgenden Befunde: erhöhte Signalintensität der Pleura, des Diaphragmas oder der Thoraxwand angrenzend an den Tumor auf den T2-gewichteten Aufnahmen, Nachweis von kontinuierlich in die Thoraxwand hinein verfolgbares Weichteilgewebe ausgehend vom Tumor in gleicher Signalintensität sowie als zuverlässigstes Zeichen, ausgehend vom Tumorgewebe eine
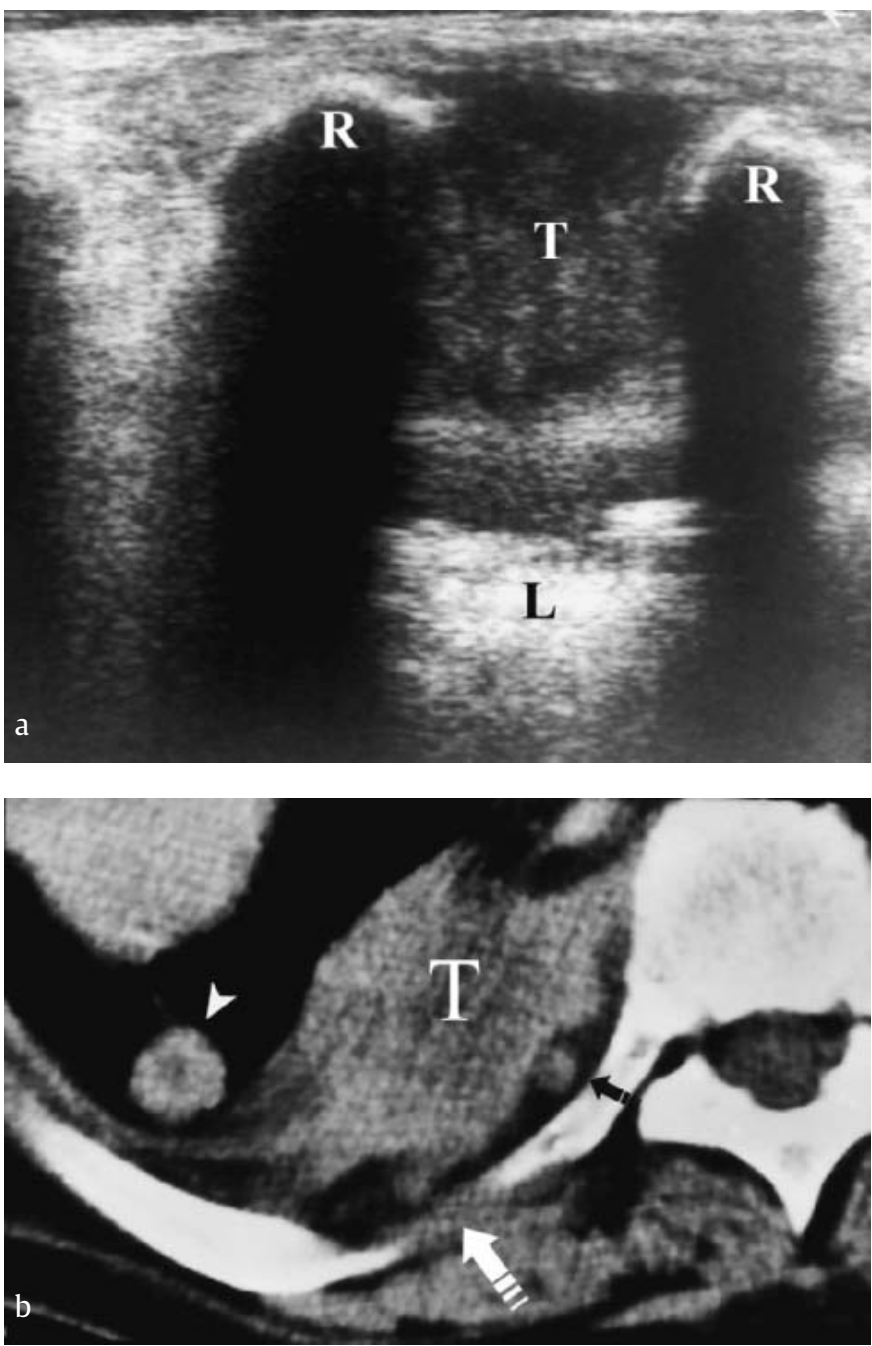

Anreicherung nach intravenöser KM-Gabe im Bereich der parietalen Pleura, des Diaphragmas oder der Thoraxwand auf T1-gewichteten Aufnahmen (Abb. 15, Abb. 16) [21,24,59]. Diese Befunde werden am besten dargestellt mit Oberflächenspulen, die eine deutlich verbesserte räumliche Auflösung bieten (Abb. 17c) [24]. Koronale und sagittale MRT-Aufnahmen zeigen exzellent die Ausdehnung von Pancoast-Tumoren und ihren Bezug zu den Anteilen des Plexus brachialis (Abb.15).

In der Mehrzahl der Fälle von Pleurabefall bei malignem Lymphom findet man entweder einen ausgedehnten Lymphknotenbefall mediastinal oder es handelt sich um ein Rezidiv. In bis zu $30 \%$ kommt es bei Hodgkin- und Non-Hodgkin-Lymphomen zu einer Pleurabeteiligung. Bei direkter Beteiligung sind meist flache breitbasig aufsitzende Lymphom-Plaques nachweisbar, häufiger beim Non-Hodgkin-Lymphom [76]. Auf Röntgenaufnahmen kann dieser Pleurabefall dem Nachweis entgehen oder im Ausmaß unterschätzt werden [77]. Der erkennbare viszerale pleurale Lymphombefall kann die parietale Pleura und die angrenzende Thoraxwand infiltrieren, dabei werden die Rippen nahezu immer ausgespart $[58,78,79]$. Lediglich maligne Lymphome, die aus einer Rippe heraus entstehen, führen zu einer ossären Destruktion derselben. Dünnschicht-CT oder -MRT sind die Methoden der Wahl zum Nachweis eines Pleurabefalls, was das Staging insbesondere beim Hodgkin-Lymphom ändern würde wie auch die

Abb. 17a-c Bronchialkarzinom-Rezidiv mit Invasion der Thoraxwand. a Sonographie des zwischen den Rippen (R) infiltrierenden Tumoranteils (T) mit hochauflösendem 7,5 MHz-Schallkopf. Schallschatten durch die Rippen, inhomogene Echodichte durch die belüftete Lunge (L). b CT-Ausschnitt im Bereich des Tumors $(T)$, zapfenartige Tumorausläufer in die Thoraxwand (Pfeile) sowie direkt benachbarte pulmonale Metastase (Pfeilspitze). c Hochaufgelöste sagittale T1-gewichtete native MRT zeigt den Tumor $(\mathrm{T})$ mit Kaudalverlagerung des intakten Zwerchfells (Pfeil) sowie zwei Tumorzapfen, die in die Thoraxwand infiltrieren (Pfeilspitzen). Rippen (R).

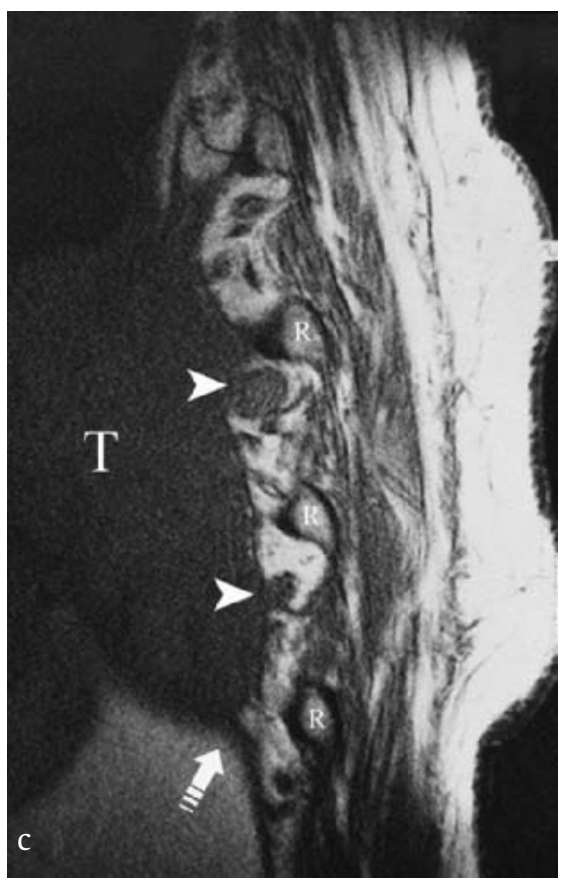




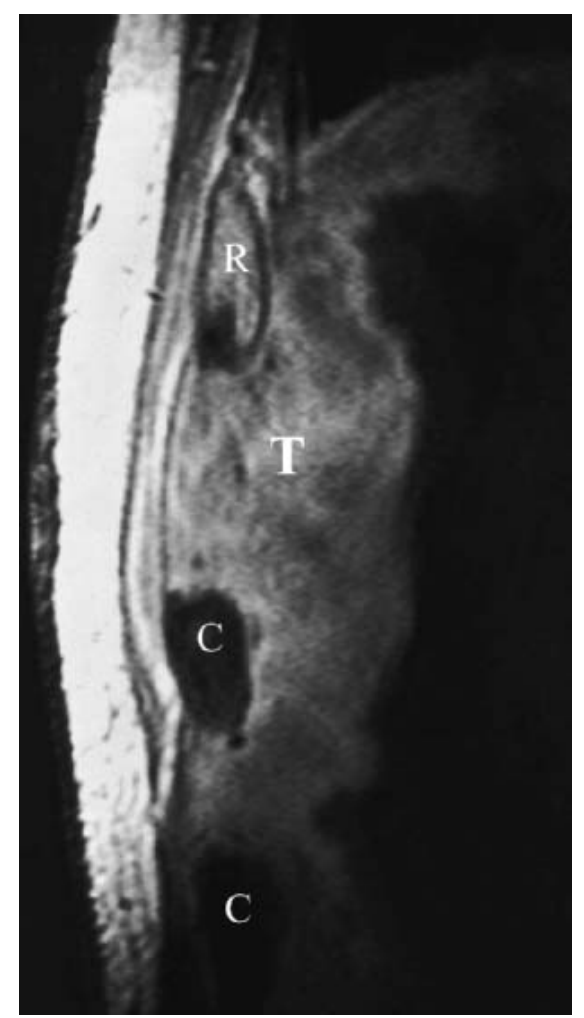

Abb. 18 Pleurales malignes Lymphom. T1-gewichtetes sagittales MRT nach KM-

Gabe durch die ventrale Thoraxwand. Kranial noch Rippenanschnitt (R), weiter kaudal Rippenknorpel (C) angeschnitten. Das ausgedehnte KM-anreichernde Tumorgewebe $(T)$ reicht bis in die Thoraxwand unterhalb des subkutanen Fettgewebes, spart jedoch die knöchernen bzw. kartilaginären Anteile der Thoraxwand aus.
Szintigraphien sind bei umschriebenen pleuralen Erkrankungen in aller Regel nicht indiziert, auch wenn immer mal wieder z.B. die Gallium-Szintigraphie beim malignen Lymphom reevaluiert wird [80]. Auch der Stellenwert der PET bei lokalisierten Pleuraerkrankungen ist bisher nicht ausreichend untersucht $[25,66,67]$. Unsere Erfahrungen beruhen wie in den bisherigen Veröffentlichungen auf Einzelfällen. Dennoch zeigte auch in diesen Fällen die PET umschriebene hohe Aktivitätsaufnahmen als Hinweis auf eine Neoplasie: ein Patient hatte ein primäres malignes Pleuramesotheliom, ein weiterer eine Pleurametastase eines bis zur PET-Untersuchung nicht entdeckten Bronchialkarzinoms, das auf den PET-Aufnahmen ebenfalls sicher diagnostiziert werden konnte (Abb. 19a, b). Auf der anderen Seite wurde in einer weiteren Einzelfallbeschreibung in der Lokalisation eines benignen Pleurafibroms ein umschriebener Bezirk erniedrigter Aktivitätsaufnahme gegenüber normal gesehen, ein Benignitäts-Kriterium [66]. Weitergehende Aussagen zum (differenzial)diagnostischen Potenzial der PET sind bei überwiegend niedrigen Fallzahlen bisher nicht möglich [25,81].

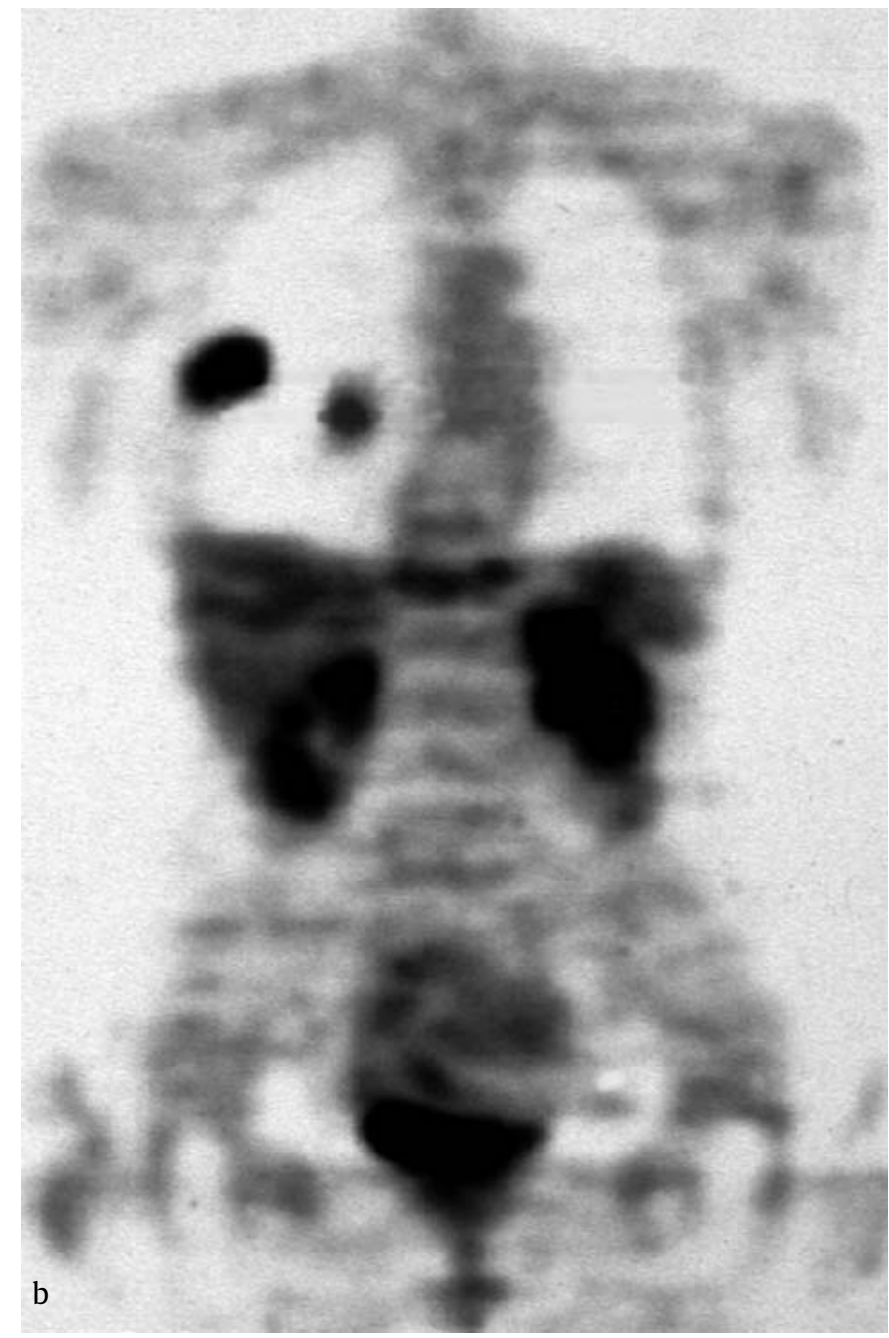

Therapie $[58,78,79]$. Während die CT pleurale knotige oder plaque-artige Veränderungen besser als die MRT abbilden kann, eignet sich die MRT besser zum Nachweis einer Beteiligung der Thoraxwand [79]. In diesen Fällen sind KM-unterstützte T1-gewichtete Aufnahmen die Methode der Wahl aufgrund der überlegenen Weichteilgewebekontrastauflösung gegenüber der CT (Abb. 18) [15, 23, 24,59]. Allerdings bietet sich die CT eher als Verfahren für die Verlaufskontrollen therapierter maligner Lymphome an, da nicht selten zystische Veränderungen oder Verkalkungen in Lymphknoten oder pleural nachweisbar sind, die der MRT meist entgehen.

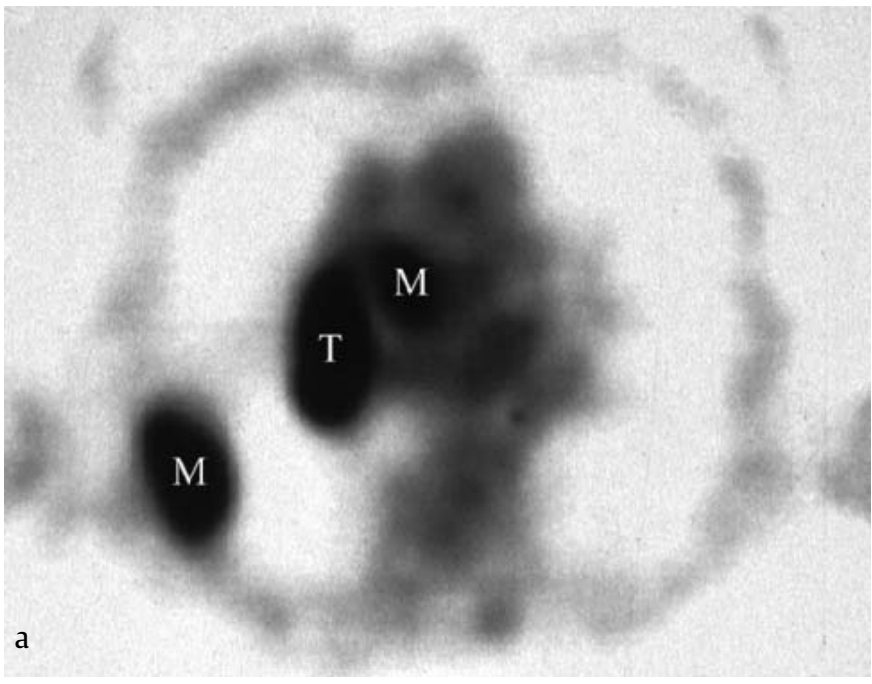

Abb. 19a, b Pleurale Raumforderung rechts unklarer Genese. a Transversale PET 120 min nach 18-FDG-Applikation zeigt vermehrte Glukoseaufnahme im Pleuraprozess (M), rechts zentral im Hilusbereich $(T)$ sowie angrenzend mediastinal (M). b Zugehöriges koronales PET, Pleuraprozess zusammen mit dem Anschnitt des rechts zentralen Prozesses abgebildet. Physiologischerweise hohe Glukose-Konzentration in Leber, Nieren und Blase. Histologische Sicherung eines bis dahin nicht bekannten Bronchialkarzinoms rechts zentral mit Pleurametastase und mediastinalen Lymphknotenmetastasen. 
Diffuse Pleuraerkrankungen

Neben der unspezifischen oder tuberkulösen Pleuritis beinhalten diffuse Pleuraerkrankungen hauptsächlich den Fibrothorax, das maligne Mesotheliom und die Pleurakarzinose.

Röntgenologisch ist eine diffuse pleurale Erkrankung zu vermuten, wenn eine ununterbrochene Pleuraverdickung über mindestens ein Viertel der kostalen Pleura nachweisbar ist, mit oder ohne Verrundung der kostophrenischen Winkel. Diese Definition wurde von McLoud u. Mitarb. 1985 geprägt und wird nach wie vor verwendet [82]. Lynch u. Mitarb. definierten eine diffuse Pleuraerkrankung im CT als diffuse pleurale Verdickung, die mehr als $8 \mathrm{~cm}$ kraniokaudal sowie mehr als $5 \mathrm{~cm}$ in der Breite misst und dabei dicker als $3 \mathrm{~mm}$ ist [83]. Dennoch werden die Definitionen der diffusen Pleuraerkrankung in der Bildgebung kontrovers diskutiert und in Veröffentlichungen gegebenenfalls neu benannt. Die ILO-Klassifikation für Pneumokoniosen insbesondere unterscheidet z.B. für Asbest-assoziierte Pleuraerkrankung „umschriebene Plaques“ und „diffuse Verdickung“ ohne weitergehende Aufschlüsselung [84]. Daher basiert die Diagnose einer diffusen Pleuraverdickung überwiegend auf radiologischen Befunden. Eine Reihe von radiologischen Hinweisen und Befunden erlaubt eine Differenzierung verschiedener Ursachen für einen Fibrothorax auf Röntgenaufnahmen und CT-Aufnahmen.

Der Nachweis von begleitender Lungenparenchymbeteiligung ist meist gegeben bei Patienten mit vorhergehender tuberkulöser Pleuritis oder unspezifischem Empyem [34]. Gleiches gilt für ausgeprägte Verkalkungen im Bereich der fibrösen Veränderungen, da z.B. bei Asbest-assoziiertem Fibrothorax Verkalkungen deutlich seltener und geringer ausgeprägt gefunden werden [85]. Der Hämatothorax, hämorrhagische Ergüsse, tuberkulöse Pleuritiden und Empyeme führen meist zu unilateralen Veränderungen mit zum Teil ausgeprägten Verkalkungen, während die Asbest-assoziierte Pleuritis überwiegend bilateral mit Plaques und/ oder diffuser Pleuraverdickung einhergeht $[15,23,86]$. Die mediastinale Pleura ist beim Fibrothorax meist nicht mitbetroffen, jedoch regelmäßig involviert beim malignen Pleuramesotheliom [87]. Der Nachweis oder Ausschluss einer Beteiligung der mediastinalen Pleura kann mit CT oder MRT erfolgen und ist hilfreich in der Differenzialdiagnostik benigner versus maligner Pleuraveränderungen $[21,88]$.

Das maligne Mesotheliom der Pleura ist eine zunehmend häufiger diagnostizierte Neoplasie. Fast alle dieser Tumoren werden bei Asbest-exponierten Patienten nachgewiesen. Die Wahrscheinlichkeit, als Asbestexponierter im Laufe des Lebens an einem malignen Mesotheliom zu erkranken, liegt immerhin bei ca. $10 \%$, die Latenzzeit wird mit bis zu 35 Jahren nach Ende der Asbestexposition angegeben [89]. Einige wenige maligne Mesotheliome sollen als Folge einer Strahlentherapie aufgetreten sein [90]. Röntgenologisch imponiert das maligne Pleuramesotheliom als irregulär und/oder nodulär konfigurierte Pleuraverdickung, in 30-95\% von ipsilateralem Pleuraerguss begleitet $[91,92]$. Oft liegt ein vollständiger hemithorakaler und zirkumferenter Pleurabefall vor, eine Beteiligung der Fissuren wird in bis zu 86\% beschrieben [92]. In seltenen Fällen kann sich das Pleuramesotheliom auch als solitärer pleuraständiger Rundherd präsentieren und ein peripheres Bronchialkarzinom imitieren [93].
Charakteristischerweise verursacht ein vorhandener Pleuraerguss beim Pleuramesotheliom keine Verlagerung des Mediastinums zur Gegenseite, im Gegenteil führt die Schrumpfung der tumorverdickten Pleura meist trotz Erguss zu einer tumorseitigen Mediastinalverziehung. Mit fortschreitender Erkrankung können Rippenarrosionen röntgenologisch nachgewiesen werden [92].

Die CT als dem Thoraxröntgen überlegenes Verfahren kann sicher den Nachweis und die Ausbreitung eines malignen Pleuramesothelioms darstellen [94-97]. Diverse Studien mit großen Fallzahlen haben CT-Kriterien für den Mesotheliom-Nachweis etabliert: diffuse und noduläre Pleuraverdickung, fissuraler Tumorbefall, Pleuraerguss, hemithorakale Schrumpfung, pleurale Verkalkungen sowie eine Infiltration der Thoraxwand mit oder ohne Rippendestruktion. In den CT-Aufnahmen werden regelmäßig peribronchiale und mediastinale Lymphknotenmetastasen nachgewiesen, selten in anderen Regionen [94, 96,97]. Leider haben Vergleiche von CT- und chirurgischen Befunden gezeigt, dass in der CT der Nachweis einer Tumorinfiltration in die Thoraxwand und das Diaphragma nicht befriedigend gelingt [95-97]. Ob das neue Mehrzeilen-Verfahren hier bessere Ergebnisse zeigen wird durch die deutlich verbesserten Rekonstruktionsaufnahmen in beliebiger Ebene, ist derzeit Gegenstand verschiedener klinischer Studien (Abb. 20, Abb. 21a). Den Nachweis eines ausgedehnten Befalls mit Beteiligung des Mediastinums, des kontralateralen Hemithorax und Übergreifen auf das Abdomen kann die CT mit großer Sicherheit führen [75,95-98]. Bei der Verlaufskontrolle von Patienten nach Tumorresektion kann die CT ein Tumorrezidiv nachweisen, bevor es röntgenologisch oder klinisch manifest wird [98].

Einige Studien zeigen ein diagnostisches Potenzial der MRT beim malignen Pleuramesotheliom $[23,24,42,57,59,75,95,97,99$, 100]. Insgesamt zeigte die MRT im Vergleich zur CT bessere Ergebnisse bei der Darstellung der Tumorausbreitung, z. B. auf koronalen und sagittalen Schichten mit höherer WeichteilgewebeKontrastauflösung (Abb. 21b). Insbesondere diese Darstellung ermöglicht eine suffiziente Diagnostik hinsichtlich eines Über-

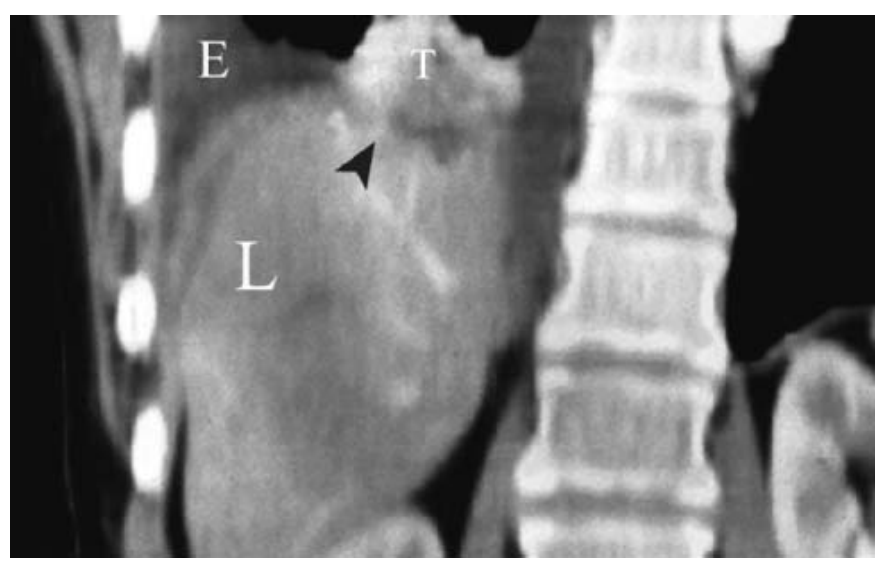

Abb. 20 Malignes Pleuramesotheliom rechts mit Übergreifen nach abdominal durch das Zwerchfell in das Leberparenchym. Koronale 2D-Rekonstruktion eines Mehrzeilen-Spiral-CT nach KM-Gabe. Gute räumliche Auflösung, Darstellung der Infiltration (Pfeilspitze) von inhomogen kontrastiertem Tumorgewebe $(T)$ von pleural durch das Zwerchfell in die Leber (L). Pleuraerguss (E). 


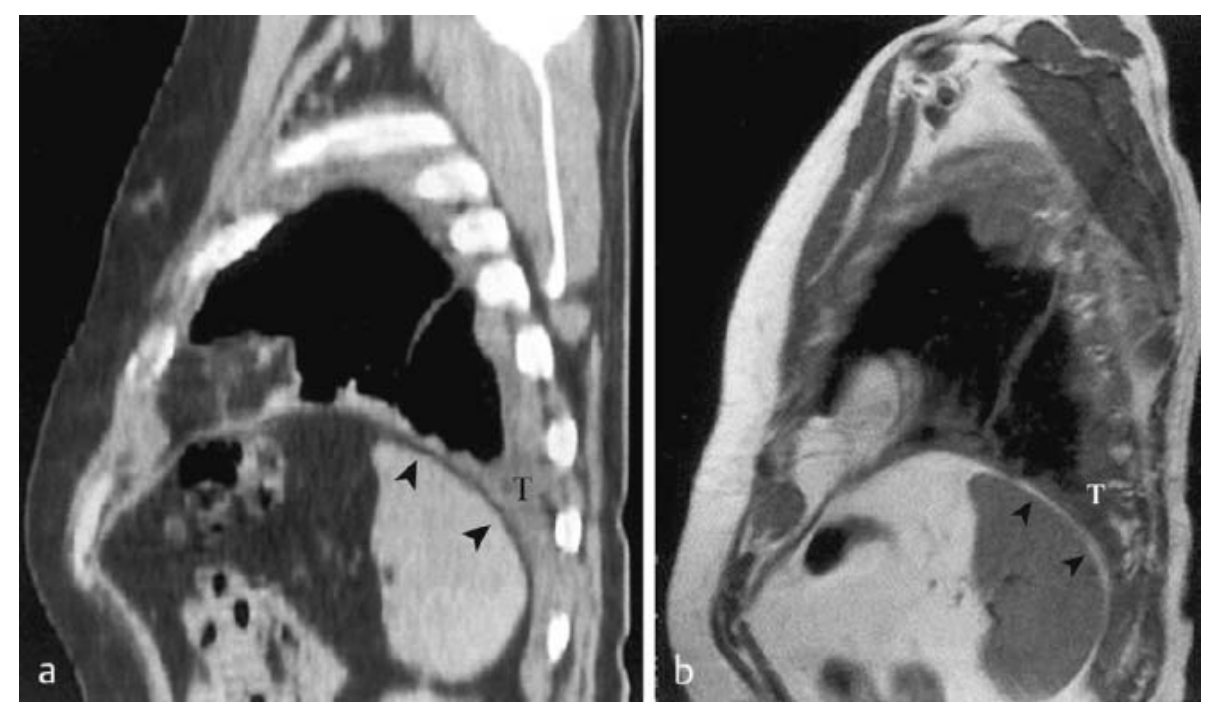

Abb. 21a, b Malignes Pleuramesotheliom links mit ausgedehntem Befall. a Mehrzeilen-Spiral-CT-2D-Rekonstruktion sagittal, Tumorgewebe ( $\mathrm{T}$ ) mit „Ausmauerung“ des Recessus ohne Zeichen des Übergreifens nach abdominal, intakte subdiaphragmale Fettlamelle (Pfeilspitzen). b Korrespondierendes natives T1-gewichtetes MRT zeigt nahezu identischen Befundaspekt mit Tumor $(T)$ weit bis in den Recessus verfolgbar, Fettlamelle subdiaphragmal intakt (Pfeilspitzen).

greifens auf Mediastinum, Thoraxwand und Diaphragma sowie nach abdominal. Die Beurteilung der Tumorresektabilität im Bereich des Mediastinums basiert auf der Intaktheit bzw. dem Infiltrationsausmaß des mediastinalen Fettgewebes um die mediastinalen Organe $[24,57,75,95,97,100]$. Dabei ist die MRT der CT überlegen, insbesondere bei der Beurteilung der Tumorausbreitung in die Thoraxwand und das Diaphragma durch die Darstellung mit KM-unterstützten T1-gewichteten Aufnahmen (vergl. Abb. 3, Abb. 9, Abb.12-18) [24,75,95]. Patienten mit resektablen

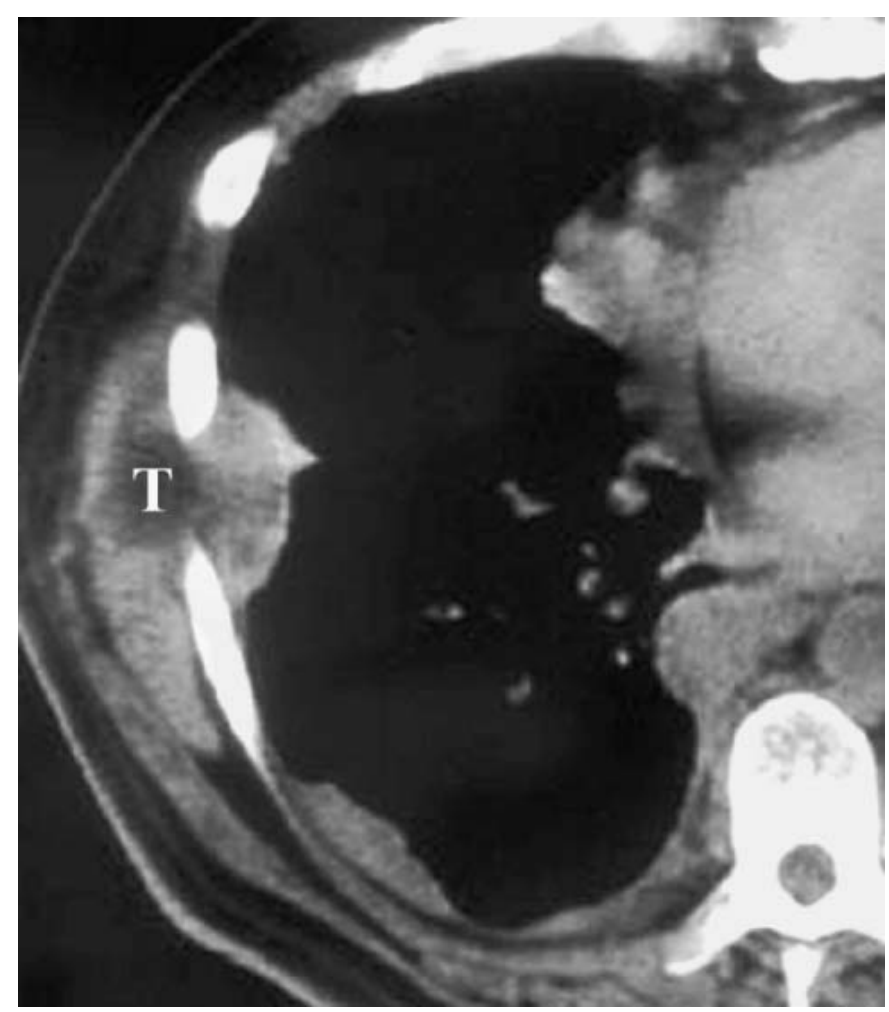

Abb. 22 Malignes Pleuramesotheliom rechts. KM-CT mit Darstellung mehrerer Tumormanifestationen pleural kostal, prävertebral und parakardial. Deutlich erkennbare Tumorinfiltration in die laterale Thoraxwand durch inhomogenes Weichteilgewebe in Kontinuität mit dem pleuralen Tumor $(\mathrm{T})$.
Tumoren zeigen eine normale extrapleurale Fettlamelle mit intakter Interkostalmuskulatur und regelrechtem Signalintensitätsmuster in der MRT. Auf CT-Aufnahmen mit schlechterem Weichteilgewebekontrast gelten als Zeichen einer Thoraxwandinfiltration eindeutig nachweisbares Tumorgewebe per continuitatem in die Thoraxwand sowie Rippendestruktionen (Abb. 22). In der MRT können innerhalb der Thoraxwand oder im Bereich des Diaphragmas schon geringgradige Signalintensitätserhöhungen auf den T2-gewichteten und KM-unterstützten T1-gewichteten Aufnahmen, die in Verbindung mit dem pleuralen tumorösen Gewebe stehen, hinweisend sein auf eine Infiltration (Abb.3). Hochauflösende MRT-Aufnahmen können im Einzelfall sogar eine beginnende tumoröse Infiltration des innersten Interkostalmuskels zeigen als frühen Nachweis eines Mesothelioms, entstanden im Bereich eines Pleuraplaques (Abb. 9) [24,100]. Nur wenige Veröffentlichungen beschäftigen sich mit dem Stellenwert der PET in der Diagnostik des malignen Pleuramesothelioms [101]. Durchgängig konnte die PET im Vergleich zur CT überlegene Ergebnisse im Nachweis sowie in der Ausbreitungsdiagnostik erzielen. Unsere Erfahrungen mit der PET beim malignen Pleuramesotheliom beruhen auf Einzelfällen, zeigen aber im Einklang mit den bisherigen Veröffentlichungen eine hohe Sensitivität im Tumornachweis (Abb. 23).

Der metastatische Befall der Pleura durch verschiedenste intraund extrathorakale Tumoren ist ursächlich für die Mehrzahl der malignen Pleuraerkrankungen. Gleichzeitig sind bei über-50Jährigen Pleurametastasen die zweithäufigste Ursache für einen Pleuraerguss nach der kardialen Insuffizienz [102,103]. Ca. 60\% aller malignen Pleuraergüsse sind ursächlich auf ein Bronchialoder Mammakarzinom zurückzuführen [103]. Bei ca. 10\% der Patienten mit malignem Pleuraerguss bleibt der Primärtumor unerkannt [102,103]. Obwohl der Pleuraerguss der bildmorphologische Hauptbefund bei der Pleurakarzinose ist, finden sich meist noch andere Veränderungen wie knotige oder rindenartige Pleuraverdickungen; Befunde wie beim Pleuramesotheliom und von diesem nicht zu differenzieren. Eine pleurale Aussaat von mehr oder weniger großen Metastasen bezieht oft auch die Fissuren und die diaphragmale Pleura mit ein (Abb. 24a-c) [102,103]. Röntgenologisch ist eine Differenzierung zum Mesotheliom oder sogar zum postinflammatorischen Fibrothorax schwierig. 
Hier können CT und MRT hilfreich sein durch Zusatzinformationen wie miterfasste Primärtumore oder die Entdeckung zusätzlicher Lungenmetastasen einer extrathorakalen Neoplasie $[15,21,23,57]$.

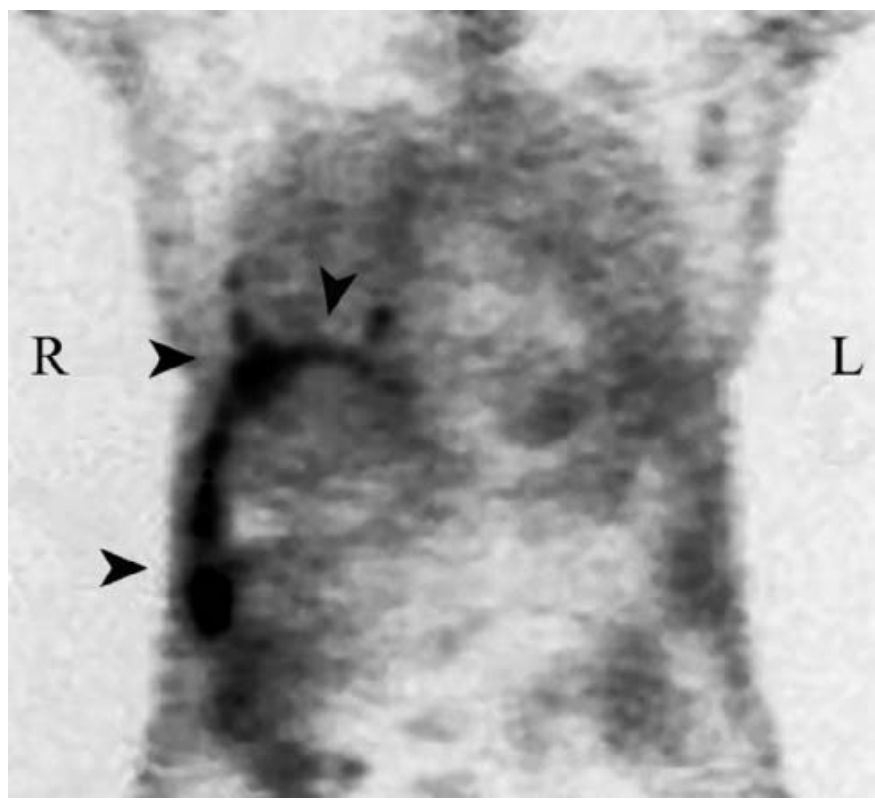

Abb. 23 Malignes Pleuramesotheliom rechts. Koronale 18-FDG-PET mit deutlicher Glukoseaufnahme im Tumorgewebe, das von basal diaphragmal weit bis in den lateralen Recessus costodiaphragmalis hineinreicht (Pfeilspitzen).

\section{Differenzialdliagnostik bei Pleuraerkrankungen}

Pathologische Veränderungen der Pleura stellen sich bildmorphologisch regelmäßig dar als unterschiedlich ausgeprägte, jedoch morphologisch sonst ähnliche Befunde: mehr oder weniger knotige Pleuraverdickungen, Verkalkungen, Plaques und Ergüsse bei sowohl benignen als auch malignen Erkrankungen. Dennoch können CT und MRT helfen, maligne und benigne Veränderungen auf der Basis von Bildbefunden zu differenzieren $[21,24,48,57,60,75,100,104]$. Die wesentlichen, auf eine maligne Genese der Erkrankungen hinweisenden Bildbefunde für beide Verfahren beinhalten: Beteiligung der gesamten hemithorakalen Pleura, zirkumferente Pleuraverdickung, knotige Pleuraverdickungen, Pleuraverdickung über $10 \mathrm{~mm}$ sowie Beteiligung der mediastinalen Pleura (Abb. 25a, b). Die Spezifität der Diagnose einer malignen Erkrankung steigt mit der Anzahl der nachweisbaren Malignitätskriterien. Der sicherste Befund für den Nachweis einer malignen Pleuraerkrankung ist die erkennbare Infiltration von Thoraxwand und/oder Diaphragma $[21,24,100]$. Hier ist die CT der MRT unterlegen durch die schlechtere Weichteilgewebe-Kontrastauflösung. Daher gelten als Methode der Wahl bei dieser Fragestellung KM-unterstützte T1-gewichtete MRT-Aufnahmen. Allerdings muss auf eine Ausnahme hingewiesen werden: die tuberkulöse Pleuritis und insbesondere das spezifische Empyem kann malignitätssuspekte Pleuraerkrankungen imitieren, einschließlich einer Beteiligung der interkostalen Thoraxwand (Abb. 26) $[21,24,100]$. Der einzige Hinweis auf eine benigne Erkrankung kann in diesen Fällen der Nachweis von pleuralen Verkalkungen sein, die häufiger bei benignen Pleuraerkrankungen gefunden werden $[15,105]$. Da CT oder MRT in der Lage sind,
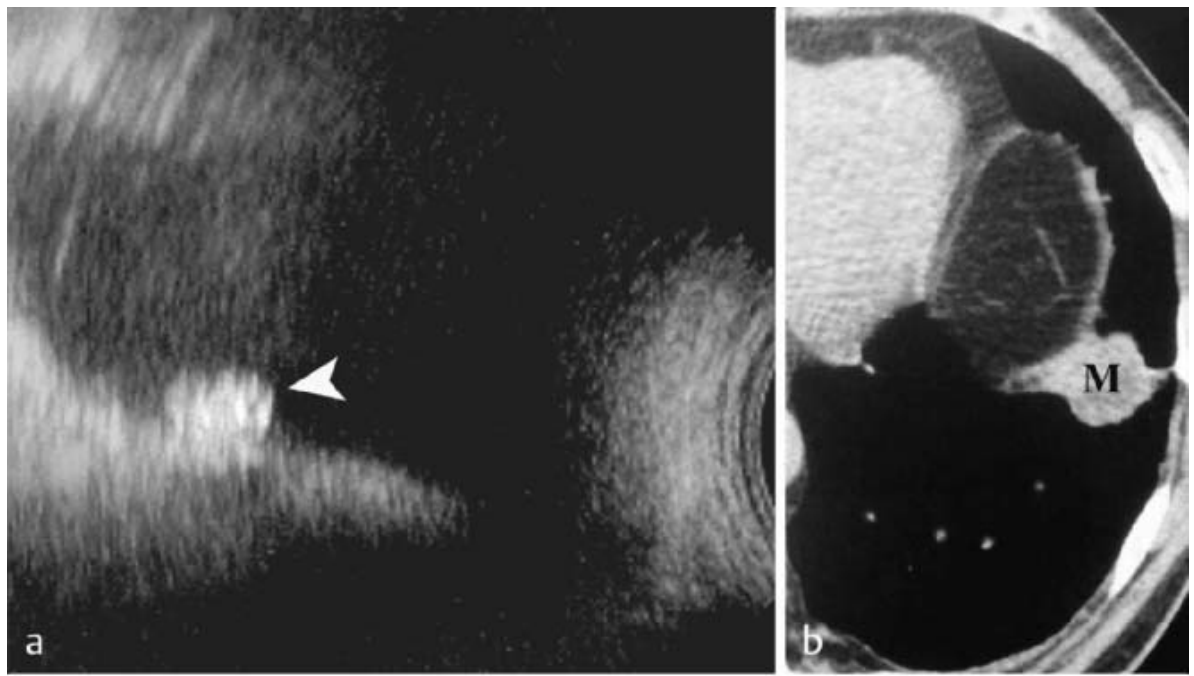

Abb. 24a-c Pleurametastase eines Chorionkarzinoms. a Bei neu aufgetretenem Pleuraerguss unklarer Genese sonographisch gut erkennbarer Prozess auf dem Zwerchfell aufsitzend (Pfeilspitze). b Transversales $C T$ zeigt nach Ergusspunktion eine kleine Raumforderung (M) auf dem Zwerchfell. c Die koronale 2D-Rekonstruktion des Mehrzeilen-Spiral-CT zeigt in der Ausschnittsvergrößerung die Metastase (M) mit Beteiligung des Diaphragmas und beginnender Ausbreitung in das subdiaphragmale Fettgewebe.

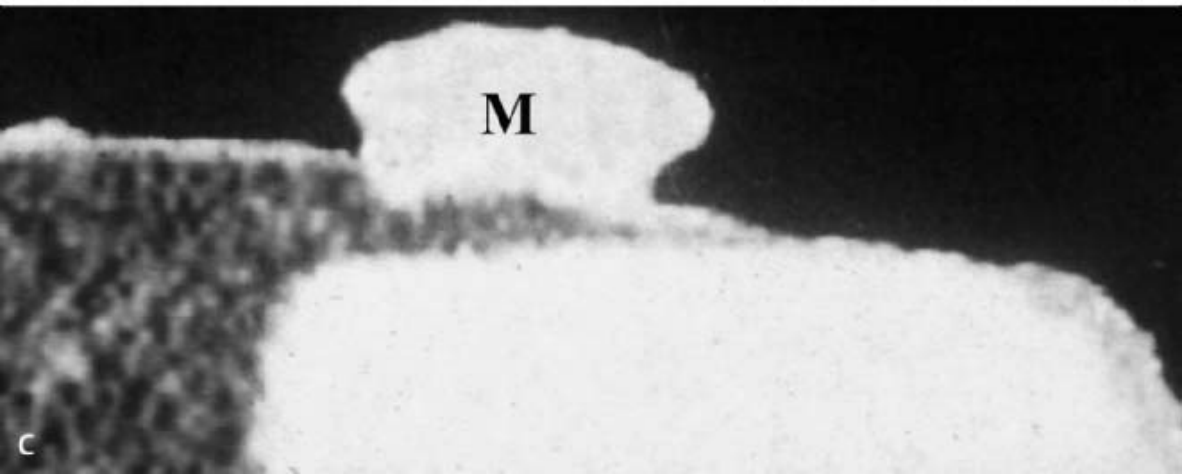




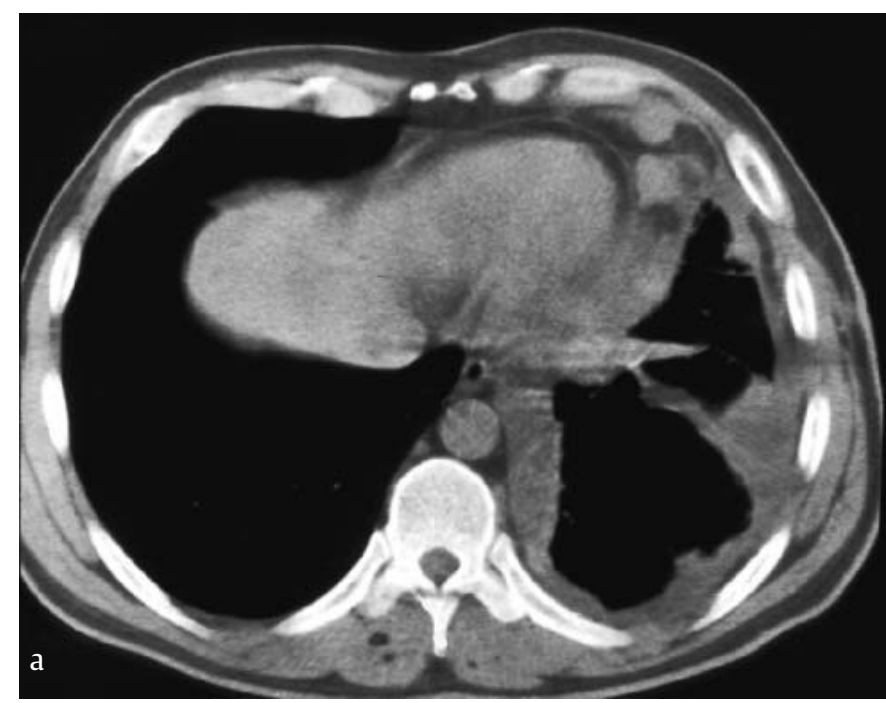

Abb. 25a, b Malignes Pleuramesotheliom, verschiedene Patienten. a Natives CT mit typischem Aspekt wie zirkumferenter Pleuraverdickung, zum Teil grob-nodulär im parakardialen Bereich, Beteiligung der Fissur und beginnender Schrumpfung des befallenen linken Hemithorax. b KM-unterstütztes T1-gewichtetes sagittales MRT bei Befall rechts hemithorakal, komplette Pleurabeteiligung einschließlich der Fissuren und des Diaphragmas sowie Auskleidung der Recessus. Intensive KM-Aufnahme, beginnende Thoraxwandbeteiligung insbesondere im Bereich des Lappenspaltansatzes möglich.

die Ausbreitung der Pleuraerkrankung übersichtlich darzustellen, können die Bildbefunde jeweils genutzt werden, um dem interventionellen Radiologen oder Thoraxchirurgen die für eine erfolgreiche Biopsie aussichtsreichste Lokalisation darzustellen. Im Falle einer durchzuführenden Thorakoskopie sollte jeweils vorher eine Schnittbildgebung mit CT oder MRT durchgeführt werden.

Die bisher durchgeführten PET-Studien bei Patienten mit Pleuraerkrankungen konnten das Potenzial der Methode bereits deutlich machen: maligne Pleuraveränderungen konnten richtig diagnostiziert werden aufgrund der deutlich erhöhten Anreicherung (vergl. Abb. 19, Abb. 23), benigne Pleuraerkrankungen wurden wegen der typischerweise reduzierten Anreicherung ebenfalls richtig als nicht-maligne eingestuft $[25,26,66,67,101]$. Weitergehende Aussagen zum Potenzial der PET bei Pleuraerkrankungen können allerdings erst durch (Multicenter-) Studien mit größeren Fallzahlen gemacht werden.

\section{Zusammenfassung}

Noch immer wird in der Mehrzahl der Fälle eine pleurale Veränderung auf der konventionellen, heute meist digital durchgeführten Röntgenaufnahme des Thorax entdeckt und vor-klassifiziert. Durch die Angrenzung an das Lungenparenchym erscheinen pleurale Raumforderungen typischerweise halbrundlich, gut abgrenzbar vom Lungenparenchym, mit scharfer Kontur und größenabhängigen Winkeln zur pleuralen Grenzfläche hin.

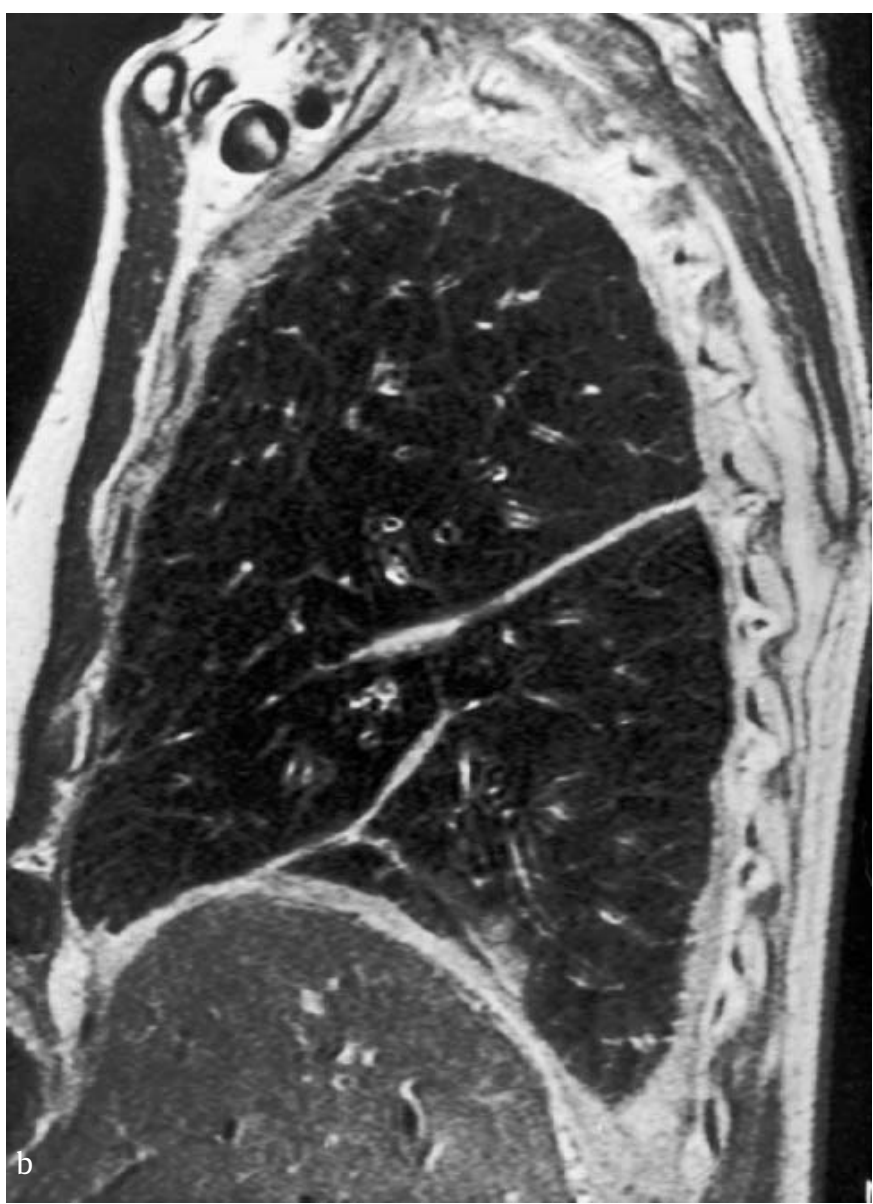

Weitere Befundkriterien beinhalten Größe und Form der Raumforderung(en) sowie deren mögliche Lageabhängigkeit, Röntgendichte, Vorhandensein und Form von Verkalkungen sowie Begleitbefunde wie z. B. Pleuraergüsse. Nach dieser Erstuntersuchung folgt in der Regel eine weitergehende Diagnostik mittels Sonographie oder CT. Da die Sonographie, obwohl exzellente Ergebnisse produzierend durch erfahrene Untersucher, nur eine eingeschränkte Untersuchung des gesamten Thoraxraums zulässt, wird die CT ganz überwiegend als weiterführendes Diagnoseverfahren der Wahl eingesetzt. Die CT kann zusätzliche Pleuraveränderungen erfassen, die mit Röntgenthoraxaufnahmen nicht nachweisbar sind. Dichtemessungen erlauben über eine Differenzierung von Flüssigkeit und Fettgewebe, Verkalkungen und Luft bereits eine weitergehende Diagnostik. Die CT kann insbesondere nach intravenöser KM-Applikation eine malignom-suspekte Infiltration von Mediastinalstrukturen, Lunge, ossären Strukturen und angrenzendem Weichteilgewebe nachweisen. Das differenzialdiagnostische Potenzial der CT basiert auf der genauen Darstellung verschiedener Pleuraveränderungen, die eher einer malignen oder benignen Genese zugeordnet werden können. Darüber hinaus stellt die CT das gesamte Ausmaß einer Pleuraerkrankung dar, kann diagnostisch relevante Nebenbefunde erfassen und hilfreich für eine Biopsieplanung sein. Der MRT kommt überwiegend die Rolle einer Problemlöserin zu bei unklarem CT-Befund. Allerdings ist die MRT aufgrund ihrer gegenüber der CT deutlich besseren Weichteilgewebe-Kontrastauflösung insbesondere nach KM-Gabe auf T1-gewichteten Aufnahmen, der primären multiplanaren Darstellung sowie der klaren Gefäßdarstellung ohne gezielte KM-Applikation als primäres di- 


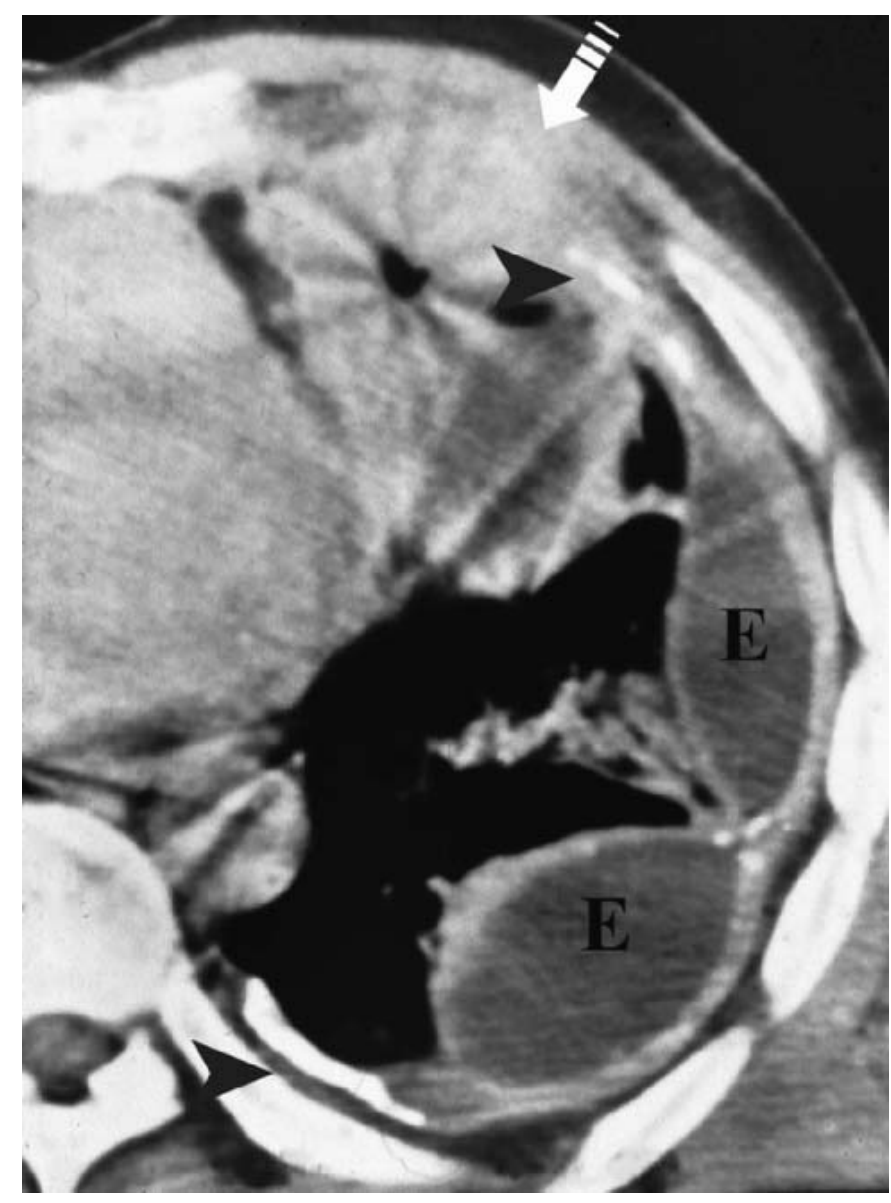

Abb. 26 Tuberkulöses Empyem mit Beteiligung der anterioren Thoraxwand. KM-CT zeigt Bild wie bei einem Asbest-induzierten malignen Pleuramesotheliom: pleurale verkalkte Plaques (Pfeilspitzen), verdickte Pleurablätter mit abgekapselten Ergussdepots (E). Übergreifen der entzündlichen Veränderungen auf die ventrale Thoraxwand (Pfeil). Histologische Sicherung eines tuberkulösen Empyems, keine Asbestexposition in der Anamnese.

agnostisches Schnittbildverfahren einsetzbar. Bei Einsatz von Oberflächenspulen ist auch die Ortsauflösung der MRT der CT vergleichbar gut. Daher eignet sich die MRT im Vergleich zur CT besonders gut für die Darstellung von Pleuraveränderungen im Bereich der Thoraxspitzen und der -basis sowie zum Nachweis einer Tumorinfiltration in die Nachbarstrukturen. T1- und T2-Signalintensitäten sowie Relaxationszeiten in der MRT sind nicht geeignet als differenzialdiagnostische Kriterien. Weder CT noch MRT können die sichernde Biopsie ersetzen. Beide Verfahren sind nicht wirklich konkurrierend, sondern ergänzen sich eher. Die CT ist preiswerter und schneller durchführbar bzw. verfügbar und wird von schwerkranken Patienten besser toleriert. Bei speziellen Fragestellungen wie Plexus-, Diaphragma- und Thoraxwandinfiltration sowie einer schweren Unverträglichkeit von Jod-haltigem CT-KM wird die MRT bevorzugt gegenüber der CT. Nuklearmedizinsche Untersuchungen bei Pleuraerkrankungen bleiben auch heutzutage die Ausnahme, allerdings könnte die PET bei weiterhin erfolgversprechenden Ergebnissen deutlich häufiger zum Einsatz kommen.

\section{Literatur}

1 Henschke CI, Davis SD, Romano RM et al. Pleural effusions: pathogenesis, radiologic evaluation, and therapy. J Thorac Imaging 1989; 4: 49

2 Collins JD, Burwell D, Furmanski S et al. Minimal detectable pleural effusions. Radiology 1972; 105: 51

3 Kocijancic I, Tercelj M, Vidmar K et al. The value of inspiratory-expiratory lateral decubitus views in the diagnosis of small pleural effusions. Clin Radiol 1999; 54: 595

4 Ruskin JA, Gurney JW, Thorsen MK et al. Detection of pleural effusions on supine chest radiographs. Am J Roentgenol 1987; 148: 681

5 Gehmacher O, Kopf A, Scheier M et al. Can pleurisy be detected with ultrasound? Ultraschall Med 1997; 18: 214

6 O'Moore PV, Mueller PR, Simeone JF et al. Sonographic guidance in diagnostic and therapeutic interventions in the pleural space. Am J Roentgenol 1987; 149: 1

7 Mathis G. Thoraxsonography - Part I: chest wall and pleura. Ultrasound Med Biol 1997; 23: 1131

8 Eibenberger KL, Dock WI, Amman ME et al. Quantification of pleural effusion: Sonography versus Radiography. Radiology 1994; 191: 681

9 Gorg C, Restrepo I, Schwerk WB. Sonography of malignant pleural effusion. Eur Radiol 1997; 7: 1195

10 Herth FJ, Becker HD. Transthoracic ultrasound. Respiration 2003; 70: 87-94

11 Civardi G, Fornari F, Cavanna L et al. Vascular signals from pleura-based lung lesions studied with pulsed Doppler ultrasonography. J Clin Ultrasound 1993; 21: 617

12 Wolek R, Mason BJ, Reeser P et al. Pleural fluid: accuracy of computed tomography in differentiating exsudates from transsudates. Conn Med 1998; 62: 259

13 Aquino SL, Webb WR, Gushiken BJ. Pleural exsudates and transsudates: diagnosis with contrast-enhanced CT. Radiology 1994; 192: 803

14 Kuhlman JE, Singha NK. Complex disease of the pleural space: radiographic and CT evaluation. RadioGraphics 1997; 17: 63

15 Müller NL. Imaging of the pleura. Radiology 1993; 186: 297

16 Naidich DP, Megibow AJ, Hilton S et al. Computed tomography of the diaphragm: peridiaphragmatic fluid localization. J Comput Assist Tomogr 1983; 7: 641

17 Halvorsen RA, Fredyshin PJ, Korobkin M et al. Ascites or pleural effusion? RadioGraphics 1986; 6: 135

18 Silverman PM, Baker ME, Mahoney BS. Atelectasis and subpulmonic fluid: a CT pitfall in distinguishing pleural from peritoneal fluid. J Comput Assist Tomogr 1985; 5: 763

19 Lawton F, Blackledge G, Johnson R. Coexistent chylous and serous pleural effusions associated with ovarian cancer: a case report of Contarini's syndrome. Eur J Surg Oncol 1985; 11: 177

20 O'Donovan PB, Eng P. Pleural changes in malignant pleural effusions: appearance on computed tomography. Cleve Clin J Med 1994; 61: 127

21 Hierholzer J, Luo L, Bittner RC et al. MRI and CT in the differential diagnosis of pleural disease. Chest 2000

22 Frola C, Cantoni S, Turtulici I et al. Transsudative vs exsudative pleural effusions: differentiation using Gd-DTPA-enhanced MRI. Eur Radiol 1997; 7: 860

23 Schmutz GR, Fisch-Ponsot C, Regent D et al. Computed tomography (CT) and magnetic resonance imaging (MRI) of pleural masses. Crit Rev Diagn Imaging 1993; 34: 309

24 Bittner RC, Schnoy N, Schoenfeld N et al. High-resolution magnetic resonance imaging (HR-MRI) of the pleura and chest wall: normal findings and pathologic changes. Fortschr Roentgenstr 1995; 162: 296

25 Alavi A, Gupta NC, Alberini JL et al. Positron emission tomography imaging in nonmalignant thoracic disorders. Semin Nucl Med 2002; 32: $293-321$

26 Gupta NC, Rogers JS, Graeber GM et al. Clinical role of F-18 fluorodeoxyglucose positron emission tomography imaging in patients with lung cancer and suspected malignant pleural effusion. Chest 2002; 122: 1918 - 1924 
27 Seow A, Kazerooni EA, Cascade PN et al. Comparison of upright inspiratory and expiratory chest radiographs for detecting pneumothoraces. Am J Roentgenol 1996; 166: 313

28 Goodman TR, Traill ZC, Phillips AJ et al. Ultrasound detection of pneumothorax. Clin Radiol 1999; 54: 736

29 Hanna JW, Reed JC, Choplin RH. Pleural infections: a clinical-radiologic review. J Thorac Imaging 1991; 6: 68

30 Levin DL, Klein JS. Imaging techniques for pleural space infections. Semin Respir Infect 1999; 14: 31

31 Spies V, Butz B, Altjohann C et al. Guidance of percutaneous biopsies, drainages, and gastrostomies with CT-fluoroscopy: comparison of puncture with conventional CT guidance and CT-fluoroscopy. Fortschr Roentgenstr 2000; 172: 374

32 Sonnenberg E van, Nakamoto SK, Mueller PR et al. CT- and ultrasound-guided catheter drainage of empyemas after chest-tube failure. Radiology 1984; 151: 349

33 Neff CC, Sonnenberg E van, Lawson DW et al. CT follow-up of empyemas: pleural peels resolve after percutaneous catheter drainage. Radiology 1990; 176: 195

34 Bressler EL, Francis IR, Glazer GM et al. Bolus contrast medium enhancement for distinguishing pleural from parenchymal lung disease: CT features. J Comput Assist Tomogr 1987; 11: 436

35 Aberle DR, Gamsu G, Ray CS. High-resolution computed tomography of asbestos-related diseases. Semin Roentgenol 1991; 26: 118

36 Al Jarad N, Poulakis N, Pearson MC et al. Assessment of asbestos-induced pleural disease by computed tomography - correlation with chest radiograph and lung function. Respir Med 1991; 85: 203

37 Ikezoe J, Takeuchi N, Kido S et al. Dynamic range control processing of digital chest images. A clinical evaluation. Acta Radiol 1996; 37: 107

38 Staples CA. Computed tomography in the evaluation of benign asbestos-related disorders. Radiol Clin North Am 1992; 30: 1191

39 Topcu F, Bayram H, Simsek M et al. High-resolution computed tomography in cases with environmental exposure to asbestos in turkey. Respiration 2000; 67: 139

40 Genevois PA, Vuyst P De, Dedeire S et al. Conventional and high-resolution CT in asymptomatic asbestos-exposed workers. Acta Radiol 1994; 35: 226

41 Im JG, Webb WR, Rosen A et al. Costal pleura: appearance at high-resolution CT. Radiology 1989; 171: 125

42 Boraschi P, Neri S, Braccini G et al. Magnetic resonance appearance of asbestos-related benign and malignant pleural diseases. Scand J Work Environ Health 1999; 25: 18

43 Lonneux M, Jamar F, Leners $\mathrm{N}$ et al. Uptake of In-111 pentetreotide by pleural plaques. Clin Nucl Med 1998; 23: 298

44 Gevenois PA, Maertelaer V de, Madani A et al. Asbestosis, pleural plaques, and diffuse pleural thickening: three distinct benign responses to asbestos exposure. Eur Respir J 1998; 11: 1021

45 Batra P, Brown K, Hayashi K et al. Rounded atelectasis. J Thorac Imaging 1996; 11: 187

46 Marchbank ND, Wilson AG, Joseph AE. Ultrasound features of folded lung. Clin Radiol 1996; 51: 433

47 Yamaguchi T, Hayashi K, Ashizawa K et al. Magnetic resonance imaging of rounded atelectasis. J Thorac Imaging 1997; 12: 188

48 Kiryu T, Ohashi N, Matsui E et al. Rounded atelectasis: delineation of enfolded visceral pleura by MRI. J Comput Assist Tomogr 2002; 26: 37-8

49 England DM, Hochholzer L, McCarthy MJ. Localized benign and malignant fibrous tumors of the pleura: a clinicopathologic review of 223 cases. Am J Surg Pathol 1989; 13: 640

50 Yang PC. Ultrasound-guided transthoracic biopsy of the chest. Radiol Clin North AM 2000; 38: 323

51 Spizarny DL, Gross BH, Shepard JO. CT findings in localized fibrous mesothelioma of the pleural fissure. J Comput Assist Tomogr 1986; 10: 942

52 Dynes MC, White EM, Fry WA et al. Imaging manifestations of pleural tumors. RadioGraphics 1992; 12: 1191

53 Kuhlman JE, Fishman EK, Berg WA et al. Evaluation of the chest wall: problem solving with CT and MR imaging. Radiology 1993; 189: 357

54 Saifuddin A, Costa P Da, Chalmers AG et al. Primary malignant localized fibrous tumors of the pleura: clinical, radiological, and pathological features. Clin Radiol 1992; 45: 13
55 Shirakawa T, Fukuda K, Miyamoto Y et al. Parietal pleural invasion of lung masses: evaluation with CT performed during deep inspiration and expiration. Radiology 1994; 192: 809

56 Perrot M de, Fischer S, Brundler MA et al. Solitary fibrous tumors of the pleura. Ann Thorac Surg 2002; 74: 285-293

57 Falashi F, Battolla L, Mascalchi M et al. Usefulness of MR signal intensity in distinguishing benign from malignant pleural disease. Am J Roentgenol 1996; 166: 963

58 Carlsen SE, Bergin CJ, Hoppe RT. MR imaging to detect chest wall and pleural involvement in patients with lymphoma: effect on radiation therapy planning. Am J Roentgenol 1993; 160: 1191

59 Bittner RC, Kaiser D, Loddenkemper R et al. Wertigkeit der Magnetresonanztomographie (MR) bei Pancoast- und anderen Tumoren mit Infiltration der Thoraxwand. Pneumologie 1990; 44: 62

60 Tateishi U, Nishihara H, Morikawa T et al. Solitary fibrous tumor of the pleura: MR appearance and enhancement pattern. J Comput Assist Tomogr 2002; 26: 174-179

61 Feretti GR, Chiles C, Choplin RH et al. Localized benign fibrous tumors of the pleura. Am J Roentgenol 1997; 169: 683

62 Padovani B, Mouroux J, Raffaelli C et al. Benign fibrous mesothelioma of the pleura: MR study and pathologic correlation. Eur Radiol 1996; 6: 425

63 Escott N, Masood K, Millward S. The diagnosis of a pleural lipoma by CT and fine-needle biopsy to avoid thoracotomy. J Can Assoc Radiol 1988; 39: 57

64 Epler GR, McLoud TC, Munn CS et al. Pleural lipoma: diagnosis by computed tomography. Chest 1986; 90: 65

65 Munk PL, Müller NL. Pleural liposarcoma: CT diagnosis. J Comput Assist Tomogr 1988; 12: 709

66 Buchmann I, Guhlmann CA, Elsner K et al. F-18-FDG-PET for primary diagnosis and differential diagnosis of pleural processes. Nuklearmedizin 1999; 38: 319

67 Lassen U, Daugaard G, Eigtved A et al. 18F-FDG whole body positron emission tomography (PET) in patients in patients unknown primary tumours (UPT). Eur J Cancer 1999; 37: 1076

68 Facciolo F, Cardillo G, Lopergolo $\mathrm{M}$ et al. Chest wall invasion in nonsmall cell lung carcinoma: a rationale for en bloc resection. J Thorac Cardiovasc Surg 2001; 121: 649-656

69 Scott IR, Müller NL, Miller RR et al. Resectable stage III lung cancer: CT, surgical, and pathologic correlation. Radiology 1988; 166: 5

70 Beute GH, Lewis JW Jr, Madrazo BL et al. Limitations of CT in evaluation of neoplasms involving the chest wall. J Comput Assist Tomogr 1987; 11: 290

71 Yokoi K, Mori K, Miyazawa N et al. Tumor invasion of the chest wall and mediastinum in lung cancer: evaluation with pneumothorax CT. Radiology 1991; 181: 147

72 Kodalli N, Erzen C, Yuksel M. Evaluation of parietal pleural invasion of lung cancers with breathhold inspiration and expiration MRI. Clin Imaging 1999; 23: 227

73 Padovani B, Mouroux J, Seksik L et al. Chest wall invasion by bronchogenic carcinoma: evaluation with MR imaging. Radiology 1993; 187: 33

74 Knisely BL, Broderick LS, Kuhlmann JE. MR imaging of the pleura and chest wall. Magn Reson Imaging Clin N Am 2000; 8: 125

75 Knuuttila A, Kivisaari L, Kivisaari A et al. Evaluation of pleural disease using MR and CT. With special reference to malignant pleural mesothelioma. Acta Radiol 2001; 42: 502-507

76 North LB, Libshitz HI, Corigan JG. Thoracic lymphoma. Radiol Clin N Am 1990; 28: 754

77 Bernardeschi P, Bonechi I, Urbano U. Recurrent pleural effusion as manifesting feature of primitive chest wall Hodgkin's disease. Chest 1988; 94: 424

78 Aquino SL, Chen MY, Kuo WT et al. The CT appearance of pleural and extrapleural disease in lymphoma. Clin Radiol 1999; 54: 647

79 Negendank WG, Alkatib A, Karanes C et al. Lymphomas: MR imaging contrast characteristics with clinical-pathologic correlations. Radiology 1990; 177: 209

80 Chajari M, Lacroix J, Peny AM et al. Gallium-67 scintigraphy in lymphoma: is there a benefit of image fusion with computed tomography? Eur J Nucl Med Mol Imaging 2002; 29: 380 - 387

81 Goldsmith SJ, Kostakoglu L. Nuclear medicine imaging of lung cancer. Radiol Clin North Am 2000; 38: 511

82 McLoud TC, Woods BO, Carrington CB et al. Diffuse pleural thickening in an asbestos-exposed population: prevalence and causes. Am J Roentgenol 1985; 144: 9 
83 Lynch DA, Gamsu G, Aberle DR. Conventional and high-resolution computed tomography in the diagnosis of asbestos-related disease. RadioGraphics 1989; 9: 523

84 International Labour Office. Guidelines for the use of the ILO international classification of radiographs of pneumoconioses, revised edition. International Labour Office Occupational Safety and Health Series, no. 22 (revised 1980). Geneva, Switzerland: International Labour Office, 1980

85 Friedman AC, Fiel SB, Radecki PD et al. Computed tomography of benign pleural and pulmonary parenchymal abnormalities related to asbestos exposure. Semin Ultrasound CT MR 1990; 11: 393

86 Aberle DR, Gamsu G, Ray CS et al. Asbestos-related pleural and parenchymal fibrosis: detection with high-resolution CT. Radiology 1988; 166: 729

87 Herbert A. Pathogenesis of pleurisy, pleural fibrosis, and mesothelial proliferation. Thorax 1986; 41: 176

88 McLoud TC. The use of CT in the examination of asbestos-exposed persons. Radiology 1988; 169: 862

89 Mossman BT, Gee JBL. Asbestos-related diseases. N Engl J Med 1989; 320: 1721

90 Kawashima A, Libshitz HI, Lukeman JM. Radiation-induced malignant pleural mesothelioma. Can Assoc Radiol J 1990; 41: 384-385

91 Ball NJ, Green FH, Kieser T et al. Pleural multicystic mesothelial proliferation. The so-called multicystic mesothelioma. Am J Surg Pathol 1990; 14: 375

92 Wechsler RJ, Steinert RM, Conant EF. Occupationally induced neoplasms of the lung and pleura. Radiol Clin North Am 1992; 30: 1245

93 Gotfried MH, Quan SF, Sobonya RE. Diffuse epithelial pleural mesothelioma presenting as a solitary lung mass. Chest 1983; 84: 99

$94 \mathrm{Ng}$ CS, Munden RF, Libshitz HI. Malignant pleural mesothelioma: the spectrum of manifestations on CT in 70 cases. Clin Radiol 1999; 54: 415
95 Layer G, Schmitteckert H, Steudel A et al. MRT, CT and sonography in the preoperative assessment of the primary tumor spread in malignant pleural mesothelioma. Rofo Fortschr Geb Rontgenstr Neuen Bildgeb Verfahr 1999; 170: 365

96 Maasilta P, Vehmas T, Kivisaari L et al. Correlations between findings at computed tomography (CT) and at thoracoscopy/thoracotomy/autopsy in pleural mesothelioma. Eur Respir J 1991; 4: 952

97 Heelan RT, Rusch VW, Begg CB et al. Staging of malignant pleural mesothelioma: comparison of CT and MR imaging. Am J Roentgenol 1999; 172: 1039

98 Adams H, Butchart EG. Computed tomographic assessment of patients following radical surgery for malignant mesothelioma. Clin Radiol 1992; 45: 120

99 Lorigan JG, Lipshitz HI. MR imaging of malignant pleural mesothelioma. J Comput Assist Tomogr 1989; 13: 617

${ }^{100}$ Bittner RC, Teichgraeber UK. Imaging techniques in the diagnosis of pleural diseases. In: Loddenkemper R, Antony VB, eds. Pleural diseases. The European Respiratory Monograph. 2002; 22: 76-109

101 Gerbaudo VH, Sugarbaker DJ, Britz-Cunningham S et al. Assessment of malignant pleural mesothelioma with (18)F-FDG dual-head gamma-camera coincidence imaging: comparison with histopathology. J Nucl Med 2002; 43: 1144-1149

102 Matthay RA, Coppage L, Shaw C et al. Malignancies metastatic to the pleura. Invest Radiol 1990; 25: 601

103 Henschke CI, Yankelevitz DF, Davis SD. Pleural disease: multimodality imaging and clinical management. Curr Probl Diagn Radiol 1991; 20: 159

104 Tateishi U, Kusumoto M, Hasegawa T et al. Primary malignant fibrous histiocytoma of the chest wall: CT and MR appearance. J Comput Assist Tomogr 2002; 26: $558-563$

${ }^{105}$ Light RW. Diseases of the pleura, mediastinum, chest wall, and diaphragm. In: George RB, Light RW, Matthay MA, Matthey RA, eds. Chest medicine. Baltimore: Williams \& Wilkins, 1990: 381 